\title{
Synthesis and Antihypertensive Screening of New Derivatives of Quinazolines Linked with Isoxazole
}

\author{
Mujeeb Ur Rahman, ${ }^{1}$ Ankita Rathore, ${ }^{1}$ Anees A. Siddiqui, ${ }^{1}$ \\ Gazala Parveen, ${ }^{2}$ and M. Shahar Yar ${ }^{1}$ \\ ${ }^{1}$ Department of Pharmaceutical Chemistry, Faculty of Pharmacy, Jamia Hamdard (Hamdard University), Hamdard Nagar, \\ New Delhi 110062, India \\ ${ }^{2}$ SunRise University, Alwar 301030, India \\ Correspondence should be addressed to M. Shahar Yar; yarmsy@rediffmail.com
}

Received 28 February 2014; Revised 11 May 2014; Accepted 13 May 2014; Published 12 June 2014

Academic Editor: Kazim Husain

Copyright (C) 2014 Mujeeb Ur Rahman et al. This is an open access article distributed under the Creative Commons Attribution License, which permits unrestricted use, distribution, and reproduction in any medium, provided the original work is properly cited.

A series of 7-substituted-3-(4-(3-(4-substitutedphenyl)-4,5-dihydroisoxazol-5-yl)phenyl)-2-substituted quinazolin-4(3H)-one (1-30) have been synthesized by the cyclization of (E)-3-(4-(3-substitutedphenyl)acrylolyl)phenyl)-2-(substitutedphenyl)-7substituted quinazolin-4-(3H)-one with hydroxylamine hydrochloride. The synthesized compounds were examined for their in vivo antihypertensive activity using albino rats. All the titled compounds exhibited good to moderate antihypertensive activity. Compounds 7-Chloro-3-(4-(3-(4-chlorophenyl)-4,5- dihydroisoxazol-5-yl)phenyl)-2-p-tolylquinazolin-4(3H)-one (23) and 7-Chloro-3-(4-(3-(4-chlorophenyl)-4,5-dihydroisoxazol-5-yl)phenyl)-2-(4-methoxyphenyl)quinazolin-4(3H)-one (24) exhibited potent antihypertensive activity through their anticipated $\alpha_{1}$-adrenergic receptor blocking property similar to its clinically used analogue, prazosin, without affecting heart rate with prolonged duration of action when tested in adrenaline induced hypertension in anaesthetized rats.

Dedicated in the memory of Professor M.S.Y Khan

\section{Introduction}

Among the major risk factors for arterial diseases, hypertension is one of the most life threatening health problems in the modern world $[1,2]$. In 2000 , the figure of total number of adults with hypertension was 972 million and this may rise by about $60 \%$ to a total of 1.56 billion by 2025 [3]. Moreover, in India, about $70 \%$ of coronary heart diseaserelated deaths occur in people younger than 70 years [4]. $\alpha_{1}$ Adrenergic receptor antagonist like prazosin $[5,6]$, terazosin, and doxazosin are reputed class of antihypertensive agents derived from quinazoline. Prazosin has selectivity to blocks postsynaptic $\alpha_{1}$-adrenergic receptors while having no effect on presynaptic $\alpha_{2}$-adrenergic receptor and is responsible for the inhibition of norepinephrine release from sympathetic nerve, and the piperazine ring is also very sensitive towards enzymatic hydroxylation, whereas doxazosin and terazosin [7] were found to possess longer duration and less reflex tachycardia than prazosin. Since quinazoline derivatives, which belong to the $\mathrm{N}$-containing heterocyclic compounds, have universal concerns due to their widely and distinct biological activities such as diuretic [8-10], antihypertensive [11], antihistaminic $[12,13]$ analgesic, and anti-inflammatory $[14,15]$ anticancer [16] and anti-HIV [17] activities, it was therefore worthwhile to study the possibility of replacing or changing in position of the labile furoylpiperazine moiety in prazosin by a more stable isoxazole group so that the antihypertensive activity of the new derivatives remains unaltered but possesses longer duration of action due to the increased stability against enzymatic degradation. In present study we have reported 30 molecules containing quinazoline and isoxazole heterocyclic ring. 
Considering the above facts and in addition to our work on antihypertensive [18], we have synthesized 7-substituted3-(4-(3-(4-substitutedphenyl)-4,5-dihydroisoxazol-5-yl) phenyl)-2-substituted quinazolin-4(3H)-one derivatives possessing antihypertensive activity with minimum side effects (Figure 1).

\section{Materials}

All the chemicals used were of laboratory grade and procured from E. Merck (Darmstadt, Germany) and S.D. Fine Chemicals (Mumbai, India). Melting points were determined by open capillary tubes in a Hicon melting point apparatus and are uncorrected. Purity of the compounds was checked by thin layer chromatography (TLC) plates (silica gel G). Iodine chamber and UV lamp were used for the visualization of TLC spots. The Fourier Transform Infrared (FT-IR) spectra were recorded on Bio-rad FTS-135 spectrophotometer using $\mathrm{KBr}$ pellets; $v_{\max }$ values are given in $\mathrm{cm}^{-1}$. The ${ }^{1} \mathrm{H}$ NMR spectra were taken on a Bruker 400 Ultrashield $(300 / 400 \mathrm{MHz})$ NMR spectrometers using dimethylsulfoxide (DMSO)-d6 solvent and tetramethylsilane (TMS) as an internal standard (chemical shift in $\delta$, ppm). ${ }^{13} \mathrm{C}$ NMR spectra were recorded on a Bruker 400 Ultrashield $(400 \mathrm{MHz})$ NMR spectrometers using dimethylsulfoxide (DMSO)-d6 solvent. Mass spectra recorded on UPLC-MS/MS (water, Q-TOF-ESI, and Mass Lyns v 4.1) mass serial no. JAA-272 (Synapt $M S-G_{1}$ ) were presented as $m / z$. Elemental analysis was carried out on $\mathrm{CHN}$ elemental (Perkin Elmer 240 analyser) using sulphanilic acid as a standard and tungsten (VI) oxide as a combusting agent. All the results of elemental analyses corresponded to the calculated values within experimental error. The reaction was monitored by thin-layer chromatography (TLC) and spots were visualized by iodine vapors or irradiation with UV light $(254 \mathrm{~nm})$.

\section{Methods}

\subsection{Chemistry}

3.1.1. Substituted 2-Benzamidobenzoic Acid (a1-a12). To the substituted anthranilic acid (2 mmol) dissolved in $10 \%$ sodium hydroxide $(10 \mathrm{~mL})$, substituted benzoyl chloride $(2.2 \mathrm{mmol})$ was added with stirring at room temperature for over $1 \mathrm{~h}$. Upon completion, reaction mixture was quenched with cold water to obtain solid residue, which was washed with dilute $\mathrm{HCl}$ followed by water and recrystallized from ethanol. Yield: 90-96\%.

\subsubsection{2-(Substituted) Phenyl-(substituted)-4H-benzo[d]} oxazin-4-one (b1-b12). A solution of substituted 2-benzamidobenzoic acid (al-a12, $2 \mathrm{mmol})$ in acetic anhydride $(10 \mathrm{~mL})$ was heated under reflux for $2 \mathrm{~h}$ and then poured into crushed ice. The solid residue thus obtained was filtered, dried, and recrystallized with ethanol; Yield is $85-90 \%$.

3.1.3. 3-(4-Acetylphenyl)-7-substituted-2-(substitutedphenyl) quinazolin-4(3H)-one (c1-c12). A mixture of substituted benzoxazine (b1-b12, $2 \mathrm{mmol}$ ) and $p$-aminoacetophenone $(2 \mathrm{mmol})$ in methanol was heated under reflux for $3 \mathrm{~h}$. The separated solid was collected by filtration, washed with water, dried, and recrystallized from ethanol.

3.1.4. (E)-3-(4-(3-Substitutedphenyl)acrylolyl)phenyl)-2-(substitutedphenyl)-7-substituted quinazolin-4-(3H)-one (d1d30). To a magnetically stirred solution of substituted quinazolinone (c1-c12, $0.01 \mathrm{mmol})$ in ethanol, substituted aldehyde $(0.01 \mathrm{mmol})$ was added and the reaction mixture was stirred for $2 \mathrm{~h}$. Upon completion of reaction, the mixture was poured on crushed ice and then acidified with dilute $\mathrm{HCl}$. A precipitate formed which was filtered, washed with water, and recrystallized with ethanol.

3.1.5. 7-Substituted-3-(4-(3-(4-substitutedphenyl-4,5-dihydroisoxazol-5-yl)phenyl)-2-substituted quinazolin-4(3H)-one (130). A mixture of (E)-3-(4-(3-substitutedphenyl)acrylolyl) phenyl)-2-(substitutedphenyl)-7-substituted quin-azolin-4(3H)-one (d1-d30, $0.01 \mathrm{~mol})$ in absolute ethanol $(25 \mathrm{~mL})$ and water; hydroxylamine hydrochloride $(0.01 \mathrm{~mol})$ was added. The reaction mixture was heated under reflux for $5 \mathrm{~h}$ in the presence of $30 \% \mathrm{KOH}$. The mixture was poured in ice water and then acidified with dilute $\mathrm{HCl}$. The resulting solid was filtered, washed with water, dried, and crystallized with ethanol.

(1) 2-Phenyl-3-(4-(3-phenyl-4,5-dihydroisoxazol-5-yl)phenyl) quinazolin-4(3H)-one (1). Yield: 78\%; m.p. 138-140 C; TLC solvent (T:E : F, $5: 4: 1) ; R_{f} 0.63$; IR $(\mathrm{KBr}) \nu_{\max }\left(\mathrm{cm}^{-1}\right): 3052$ (Ar-CH), 1695 (CO), $1630(\mathrm{C}=\mathrm{N}), 1608,1455(\mathrm{C}=\mathrm{C}), 1220$ (C$\mathrm{O}-\mathrm{N}), 1115(\mathrm{C}-\mathrm{N}) ;{ }^{1} \mathrm{H}$ NMR (DMSO-d6) $\delta$ (ppm); 3.80 (d, $\left.2 \mathrm{H}, J=8.8 \mathrm{~Hz}, \mathrm{CH}_{\text {isox. }}\right), 5.88\left(\mathrm{dd}, 1 \mathrm{H}, J=5.5,8.4 \mathrm{~Hz}, \mathrm{CH}_{2 \text { isox. }}\right.$ ), $8.04(\mathrm{~d}, 2 \mathrm{H}, J=9.4 \mathrm{~Hz}, \mathrm{Ar}-\mathrm{H}), 8.06(\mathrm{~d}, 4 \mathrm{H}, J=7.0 \mathrm{~Hz}, \mathrm{Ar}-$ $\mathrm{H}), 8.10(\mathrm{~d}, 2 \mathrm{H}, J=6.8 \mathrm{~Hz}, \mathrm{Ar}-\mathrm{H}), 8.12(\mathrm{t}, 2 \mathrm{H}, J=5.2 \mathrm{~Hz}, J=$ $3.6 \mathrm{~Hz}, \mathrm{Ar}-\mathrm{H}), 8.14$ (d, $2 \mathrm{H}, J=8.8 \mathrm{~Hz}, \mathrm{Ar}-\mathrm{H}), 8.16$ (d, 2H, $=10 \mathrm{~Hz}, \mathrm{Ar}-\mathrm{H}$ ), 8.15-8.17 (m, 4H, Ar-H); ${ }^{13} \mathrm{C}$ NMR (DMSOd6) $\delta$; 128.5 (2C), 128.8 (2C), 131.8, 132.5 (phenyl), 43.7, 85.5, 155.7 (3C, isoxazole), 128.5 (2C), 128.8 (2C), 130.2, 131.3 (ArC), 124.4 (2C), 128.5 (2C), 132.45, 137.66 (Ar-C), 120.6, 126.6, $128.2,133.8,135.2,148.3,156.9$ (quinazoline), $160.4(1 \mathrm{C}, \mathrm{C}=\mathrm{O})$; $(m / z): 443.49\left(\mathrm{M}^{+}\right)$; \% Anal. Cal. for $\mathrm{C}_{29} \mathrm{H}_{21} \mathrm{~N}_{3} \mathrm{O}_{2}$ : C: 78.54, H: 4.77, N: 9.47. Found: C: 78.50, H: 4.72, N: 9.46.

(2) 2-(2-Chlorophenyl)-3-(4-(3-Phenyl-4,5-dihydroisoxazol-5yl)phenyl)quinazolin-4(3H)-one (2). Yield: 84\%; m.p. 140$142^{\circ} \mathrm{C}$; TLC solvent (T: E: F, $\left.5: 4: 1\right) ; R_{f} 0.66 ; \mathrm{IR}\left(\mathrm{KBr}, \mathrm{cm}^{-1}\right)$ : 3022 (Ar-CH), 1685 (CO), $1610(\mathrm{C}=\mathrm{N}), 1598,1463$ (C=C), 1230 $(\mathrm{C}-\mathrm{O}-\mathrm{N}), 1110(\mathrm{C}-\mathrm{N}), 710(\mathrm{C}-\mathrm{Cl}) ;{ }^{1} \mathrm{H}$ NMR $\left(\mathrm{DMSO}-d_{6}\right) \delta$; $3.90\left(\mathrm{~d}, 2 \mathrm{H}, J=7.6 \mathrm{~Hz}, \mathrm{CH}_{2 \text { isox. }}\right), 6.02(\mathrm{dd}, 1 \mathrm{H}, J=6.8,2.8 \mathrm{~Hz}$, $\left.\mathrm{CH}_{\text {isox. }}\right), 8.06(\mathrm{~d}, 2 \mathrm{H}, \mathrm{J}=11.6 \mathrm{~Hz}, \mathrm{Ar}-\mathrm{H}), 8.06-8.09(\mathrm{~m}, 3 \mathrm{H}$, Ar-H), 8.10 (d, 2H, J = 2.8 Hz, Ar-H), $8.12(\mathrm{t}, 2 \mathrm{H}, J=3.2, J=$ $5.6 \mathrm{~Hz}, \mathrm{Ar}-\mathrm{H}), 8.14$ (d, 2H, J = 7.2 Hz, Ar-H), 8.15 (d, 2H, J = 11.6 Hz, Ar-H), 8.16-8.18 (m, 4H, Ar-H); ${ }^{13} \mathrm{C}$ NMR (DMSO$\left.d_{6}\right) \delta ; 128.2$ (2C), 129.2 (2C), 130.8, 131.2 (phenyl), 42.2, 83.4, 156.5 (3C, isoxazole), 123.5, 126.5, 128.8, 130.2, 131.3, 132.3 (ArC), 125.8 (2C), 128.2 (2C), 132.5, 138.3 (Ar-C), 120.4, 127.7, $128.8,134.2$, 135.6, 147.6, 155.4 (quinazoline), 162.3 (1C, $\mathrm{C}=\mathrm{O})$; 


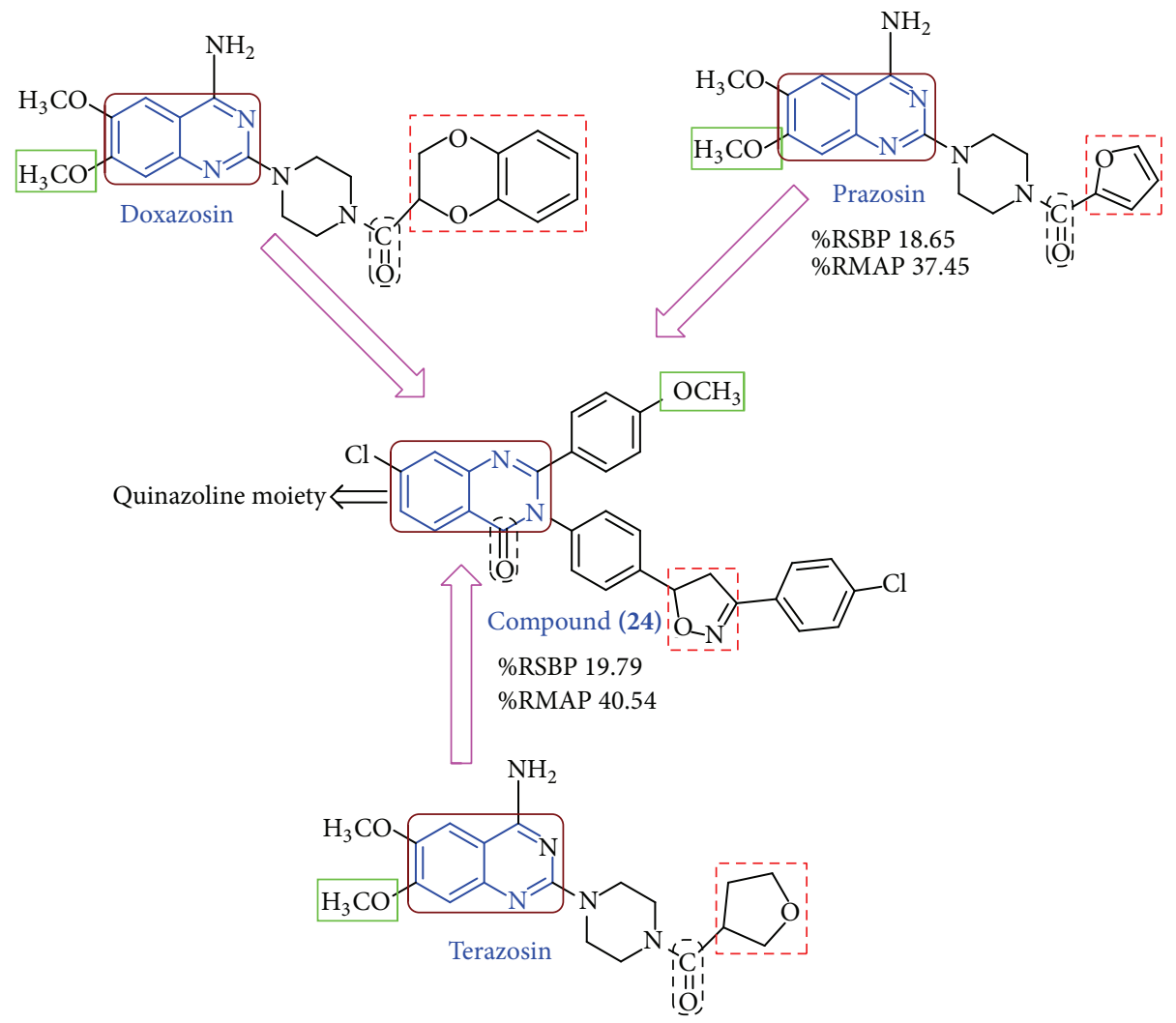

FIGURE 1: Chemical structure of active chemotherapeutic antihypertensive agents (prazosin, terazosin, and doxazosin) and rationally designed template for targeted compound (24). RSBP (rise in systolic blood pressure), RMAP (rise in mean atrial blood pressure).

\% Anal. Cal. for $\mathrm{C}_{29} \mathrm{H}_{20} \mathrm{ClN}_{3} \mathrm{O}_{2} ; \mathrm{C}, 72.88 ; \mathrm{N}, 8.79 ; \mathrm{H}, 4.22$. Found; C, 72.86; N, 8.77; H, 4.20; $\mathrm{Ms}(\mathrm{m} / z): 477.94\left(\mathrm{M}^{+}\right)$.

(3) 3-(4-(3-Phenyl-4,5-dihydroisoxazol-5-yl)phenyl)-2-p-tolylquinazolin-4(3H)-one (3). Yield: 70\%; m.p. 162-164 C; TLC solvent (T:E: F, $5: 4: 1) ; R_{f}$ 0.63; IR $\left(\mathrm{KBr}, \mathrm{cm}^{-1}\right)$ : 3012 (Ar$\mathrm{CH}), 1690$ (CO), $1634(\mathrm{C}=\mathrm{N}), 1618,1465(\mathrm{C}=\mathrm{C}), 1225$ (C-O$\mathrm{N}), 1123(\mathrm{C}-\mathrm{N}) ;{ }^{1} \mathrm{H}$ NMR (DMSO-d6) $\delta$ (ppm); 2.23 (s, 3H, $\left.\mathrm{CH}_{3}\right), 3.92\left(\mathrm{~d}, 2 \mathrm{H}, J=8.4 \mathrm{~Hz}, \mathrm{CH}_{2 \text { isox. }}\right), 6.00$ (dd, $1 \mathrm{H}, J=$ 2.8, 7.2 Hz, $\mathrm{CH}_{\text {isox. }}$ ), 8.03-8.06 (m, 5H, Ar-H), 8.08 (d, $2 \mathrm{H}$, $J=4 \mathrm{~Hz}, \mathrm{Ar}-\mathrm{H}), 8.08-8.09$ (m, 2H, Ar-H), $8.12(\mathrm{~d}, 2 \mathrm{H}, J=$ $7.6 \mathrm{~Hz}, \mathrm{Ar}-\mathrm{H}), 8.15$ (d, 2H, $J=8.4 \mathrm{~Hz}, \mathrm{Ar}-\mathrm{H}), 8.16-8.17(\mathrm{~m}$, $4 \mathrm{H}, \mathrm{Ar}-\mathrm{H}) ;{ }^{13} \mathrm{C}$ NMR (DMSO-d6) $\delta ; 20.5\left(1 \mathrm{C}, \mathrm{CH}_{3}\right), 128.5$ (2C), 128.8 (2C), 131.0, 132.6 (phenyl), 40.8, 84.7, 157.2 (3C, isoxazole), 125.2, 129.5 (2C), 130.8 (2C), 140.6 (Ar-C), 126.2 (2C), 128.8 (2C), 131.2, 137.5 (Ar-C), 120.8, 126.4, 128.8, 133.6, 136.3, 146.5, 156.4 (quinazoline), 160.6 (1C, $\mathrm{C}=\mathrm{O}$ ); \% Anal. Cal. for $\mathrm{C}_{30} \mathrm{H}_{23} \mathrm{~N}_{3} \mathrm{O}_{2} ; \mathrm{C}, 78.75 ; \mathrm{N}, 9.18 ; \mathrm{H}$, 5.07. Found; $\mathrm{C}$, 78.76; N, 9.18; H, 5.05; Ms $(m / z): 457.52\left(\mathrm{M}^{+}\right)$.

(4) 2-(4-Methoxyphenyl)-3-(4-(3-phenyl-4,5-dihydroisoxazol5-yl)phenyl)quinazolin-4(3H)-one (4). Yield: 65\%; m.p. 135$137^{\circ} \mathrm{C}$; TLC solvent (T: E : F, $\left.5: 4: 1\right) ; R_{f} 0.67 ; \mathrm{IR}\left(\mathrm{KBr}, \mathrm{cm}^{-1}\right)$ : 3022 (Ar-CH), 1675 (CO), $1625(\mathrm{C}=\mathrm{N}), 1588,1425$ (C=C), 1310 (C-O), $1226(\mathrm{C}-\mathrm{O}-\mathrm{N}), 1110(\mathrm{C}-\mathrm{N}) ;{ }^{1} \mathrm{H}$ NMR (DMSO-d6) $\delta$ $(\mathrm{ppm}) ; 2.70\left(\mathrm{~s}, 3 \mathrm{H}, \mathrm{OCH}_{3}\right), 3.80\left(\mathrm{~d}, 2 \mathrm{H}, J=8.0 \mathrm{~Hz}, \mathrm{CH}_{2 \text { isox. }}\right)$, $5.78\left(\mathrm{~d}, 1 \mathrm{H}, J=12.4 \mathrm{~Hz}, \mathrm{CH}_{\text {isox. }}\right.$ ), 8.02-8.04 (m, 5H, Ar-H), $8.07(\mathrm{~d}, 2 \mathrm{H}, J=8.4 \mathrm{~Hz}, \mathrm{Ar}-\mathrm{H}), 8.09(\mathrm{~d}, 2 \mathrm{H}, J=3.2 \mathrm{~Hz}$, Ar$\mathrm{H}), 8.11-8.20$ (m, 8H, Ar-H); ${ }^{13} \mathrm{C}$ NMR (DMSO-d6) $\delta ; 52.6$ $\left(1 \mathrm{C}, \mathrm{OCH}_{3}\right), 128.5$ (2C), 129.8 (2C), 130.2, 131.5 (phenyl), 42.0, 84.2, 155.7 (3C, isoxazole), 118.4 (2C), 120.5, 132.4 (2C), 158.4 (Ar-C), 124.4 (2C), 126.8 (2C), 131.4, 138.0 (Ar-C), 120.8, 127.6, 128.2, 133.6, 135.6, 148.3, 156.0 (quinazoline), 160.2 (1C, $\mathrm{C}=\mathrm{O}$ ); \% Anal. Cal. for $\mathrm{C}_{30} \mathrm{H}_{23} \mathrm{~N}_{3} \mathrm{O}_{3} ; \mathrm{C}, 76.09 ; \mathrm{N}, 8.87 ; \mathrm{H}, 4.90$. Found; C, 76.08; N, 8.87; H, 4.92; Ms (m/z): $473.52\left(\mathrm{M}^{+}\right)$.

(5) 7-Chloro-2-phenyl-3-(4-(3-phenyl-4,5-dihydroisoxazol-5yl)phenyl)quinazolin-4(3H)-one (5). Yield: 80\%; m.p. 166$168^{\circ} \mathrm{C}$; TLC solvent (T: E : F, $\left.5: 4: 1\right) ; R_{f} 0.54 ; \mathrm{IR}\left(\mathrm{KBr}, \mathrm{cm}^{-1}\right)$ : 3062 (Ar-CH), 1680 (CO), $1650(\mathrm{C}=\mathrm{N}), 1618,1465(\mathrm{C}=\mathrm{C})$, $1226(\mathrm{C}-\mathrm{O}-\mathrm{N}), 1122(\mathrm{C}-\mathrm{N}), 720(\mathrm{C}-\mathrm{Cl}) ;{ }^{1} \mathrm{H}$ NMR (DMSOd6) $\delta(\mathrm{ppm}) ; 3.83\left(\mathrm{~d}, 2 \mathrm{H}, J=6.6 \mathrm{~Hz}, \mathrm{CH}_{2 \text { isox. }}\right), 5.88(\mathrm{dd}, 1 \mathrm{H}$, $\left.J=4.5, J=7.7 \mathrm{~Hz}, \mathrm{CH}_{\text {isox }}\right), 7.98-8.00(\mathrm{~m}, 5 \mathrm{H}, \mathrm{Ar}-\mathrm{H}), 8.04$ $(\mathrm{d}, 2 \mathrm{H}, J=5.5 \mathrm{~Hz}, \mathrm{Ar}-\mathrm{H}), 8.06(\mathrm{~d}, 2 \mathrm{H}, J=8.8 \mathrm{~Hz}, \mathrm{Ar}-\mathrm{H})$, $8.08(\mathrm{~d}, 2 \mathrm{H}, J=9.4 \mathrm{~Hz}, \operatorname{Ar}-\mathrm{H}), 8.12(\mathrm{~d}, 2 \mathrm{H}, J=3.8 \mathrm{~Hz}, \mathrm{Ar}-$ $\mathrm{H}), 8.14-8.16$ (m, 4H, Ar-H); ${ }^{13} \mathrm{C}$ NMR (DMSO-d6) $\delta ; 128.5$ (2C), 129.0 (2C), 131.4, 132.5 (phenyl), 42.6, 84.6, 155.0 (3C, isoxazole), 128.4 (2C), 128.6, 128.8 (2C), 132.5 (Ar-C), 125.2 (2C), 128.6 (2C), 131.0, 135.8 (Ar-C), 118.5, 122.5, 127.6, 130.8, 137.8, 152.4, 156.2 (quinazoline), 161.5 (1C, C=O); \% Anal. Cal. for $\mathrm{C}_{29} \mathrm{H}_{20} \mathrm{ClN}_{3} \mathrm{O}_{2} ; \mathrm{C}, 72.88 ; \mathrm{N}, 8.79 ; \mathrm{H}, 4.22$. Found; C, 72.86; $\mathrm{N}, 8.77 ; \mathrm{H}, 4.20$; Ms $(\mathrm{m} / z)$ : $478.94(\mathrm{M}+1)$.

(6) 7-Chloro-2-(2-chlorophenyl)-3-(4-(3-phenyl-4,5-dihydroisoxazol-5-yl)phenyl)quinazolin-4(3H)-one (6). Yield: 85\%; 
m.p. $130-132^{\circ} \mathrm{C}$; TLC solvent (T:E:F, $5: 4: 1$ ); $R_{f}$ 0.65; IR $\left(\mathrm{KBr}, \mathrm{cm}^{-1}\right): 3034(\mathrm{Ar}-\mathrm{CH}), 1710(\mathrm{CO}), 1660(\mathrm{C}=\mathrm{N}), 1598$, $1480(\mathrm{C}=\mathrm{C}), 1224(\mathrm{C}-\mathrm{O}-\mathrm{N}), 1176(\mathrm{C}-\mathrm{N}), 750,610(\mathrm{C}-\mathrm{Cl}) ;{ }^{1} \mathrm{H}$ NMR (DMSO-d6) $\delta$ (ppm); $3.94\left(\mathrm{~d}, 2 \mathrm{H}, J=6.9 \mathrm{~Hz}, \mathrm{CH}_{2 \text { isox. }}\right.$ ), $5.86\left(\mathrm{dd}, 1 \mathrm{H}, J=1.2,6.9 \mathrm{~Hz}, \mathrm{CH}_{\text {isox. }}\right), 8.03-8.06(\mathrm{~m}, 5 \mathrm{H}, \mathrm{Ar}-$ $\mathrm{H}), 8.06-8.08(\mathrm{~m}, 3 \mathrm{H}, \mathrm{Ar}-\mathrm{H}), 8.10(\mathrm{~d}, 1 \mathrm{H}, J=5.4 \mathrm{~Hz}, \mathrm{Ar}-\mathrm{H})$, $8.14(\mathrm{~d}, 2 \mathrm{H}, J=0.6 \mathrm{~Hz}, \operatorname{Ar}-\mathrm{H}), 8.17(\mathrm{~d}, 2 \mathrm{H}, J=3.6 \mathrm{~Hz}, \mathrm{Ar}-$ H), 8.20-8.22 (m, 3H, Ar-H); ${ }^{13}$ C NMR (DMSO-d6) $\delta$; 128.2 (2C), 130.8 (2C), 131.4, 132.3 (phenyl), 45.7, 88.5, 154.7 (3C, isoxazole), 120.6, 124.8, 130.2, 132.3 (2C), 134.5 (Ar-C), 125.8 (2C), 127.8 (2C), 132.4, 137.6 (Ar-C), 118.3, 120.6, 128.2, 130.8, 138.25, 151.3, 155.9 (quinazoline), $162.4(1 \mathrm{C}, \mathrm{C}=\mathrm{O}) ; \%$ Anal. Cal. for $\mathrm{C}_{29} \mathrm{H}_{19} \mathrm{Cl}_{2} \mathrm{~N}_{3} \mathrm{O}_{2} ; \mathrm{C}, 67.98 ; \mathrm{N}, 8.20 ; \mathrm{H}, 3.74$. Found; C, 67.96; N, 8.22; H, 3.72; Ms $(\mathrm{m} / z)$ : $512.38(\mathrm{M}+1)$.

(7) 7-Chloro-3-(4-(3-phenyl-4,5-dihydroisoxazol-5-yl)phenyl) -2-p-tolylquinazolin-4(3H)-one (7). Yield: 70\%; m.p. 164$166^{\circ} \mathrm{C}$; TLC solvent (B : A, $\left.9: 1\right) ; R_{f} 0.67 ; \mathrm{IR}\left(\mathrm{KBr}, \mathrm{cm}^{-1}\right): 3012$ (Ar-CH), $1690(\mathrm{CO}), 1644(\mathrm{C}=\mathrm{N}), 1602,1445(\mathrm{C}=\mathrm{C}), 1225$ (C-O-N), $1154(\mathrm{C}-\mathrm{N}), 720(\mathrm{C}-\mathrm{Cl}) ;{ }^{1} \mathrm{H}$ NMR (DMSO-d6) $\delta$ (ppm); 2.32 (s, $\left.3 \mathrm{H}, \mathrm{CH}_{3}\right), 3.96\left(\mathrm{~d}, 2 \mathrm{H}, J=6.7 \mathrm{~Hz}, \mathrm{CH}_{2 \text { isox. }}\right.$ ), $5.80\left(\mathrm{dd}, 1 \mathrm{H}, J=7.8,7.0 \mathrm{~Hz}, \mathrm{CH}_{\text {isox. }}\right.$ ), $8.00-8.02(\mathrm{~m}, 5 \mathrm{H}, \mathrm{Ar}-$ $\mathrm{H}), 8.04(\mathrm{~d}, 2 \mathrm{H}, J=7.8 \mathrm{~Hz}, \mathrm{Ar}-\mathrm{H}), 8.07(\mathrm{~d}, 2 \mathrm{H}, J=9.2 \mathrm{~Hz}$, Ar-H), 8.10 (d, $2 \mathrm{H}, J=8.4 \mathrm{~Hz}, \mathrm{Ar}-\mathrm{H}), 8.12(\mathrm{~d}, 2 \mathrm{H}, J=9.2 \mathrm{~Hz}$, Ar-H), 8.11-8.18 (m, 3H, Ar-H); ${ }^{13}$ C NMR (DMSO-d6) $\delta ; 20.4$ (1C, $\mathrm{CH}_{3}$ ), 128.5 (2C), 129.8 (2C), 130.2, 131.8 (phenyl), 42.6, 84.2, 154.3 (3C, isoxazole), 125.5, 128.5 (2C), 130.3 (2C), 138.9 (Ar-C), 125.2 (2C), 127.5 (2C), 131.8, 137.2 (Ar-C), 118.8, 122.6, 130.6, 134.8, 137.4, 153.4, 156.7 (quinazoline), 160.2 (1C, $\mathrm{C}=\mathrm{O})$; \% Anal. Cal. for $\mathrm{C}_{30} \mathrm{H}_{22} \mathrm{ClN}_{3} \mathrm{O}_{2} ; \mathrm{C}, 73.24 ; \mathrm{N}, 8.54 ; \mathrm{H}, 4.51$. Found; C, 73.23; N, 8.52; H, 4.50; Ms $(\mathrm{m} / z)$ : $491.96\left(\mathrm{M}^{+}\right)$.

(8) 7-Chloro-2-(4-methoxyphenyl)-3-(4-(3-phenyl-4,5-dihydroisoxazol-5-yl)phenyl)quinazolin-4(3H)-one (8). Yield: 85\%; m.p. 170-172 ${ }^{\circ}$; TLC solvent (B:A, 9:1); $R_{f} 0.70$; IR $\left(\mathrm{KBr}, \mathrm{cm}^{-1}\right)$ : $3022(\mathrm{Ar}-\mathrm{CH}), 1693(\mathrm{CO}), 1610(\mathrm{C}=\mathrm{N}), 1588$, $1463(\mathrm{C}=\mathrm{C}), 1295(\mathrm{C}-\mathrm{O}), 1233(\mathrm{C}-\mathrm{O}-\mathrm{N}), 1122(\mathrm{C}-\mathrm{N}), 722$ (C-Cl); ${ }^{1} \mathrm{H}$ NMR (DMSO-d6) $\delta(\mathrm{ppm}) ; 2.75\left(\mathrm{~s}, 3 \mathrm{H}, \mathrm{OCH}_{3}\right)$, $3.94\left(\mathrm{~d}, 2 \mathrm{H}, J=6.9 \mathrm{~Hz}, \mathrm{CH}_{2 \mathrm{isox}}\right), 5.82(\mathrm{dd}, 1 \mathrm{H}, J=2.8, J=$ $\left.8.0 \mathrm{~Hz}, \mathrm{CH}_{\text {isox. }}\right), 8.04-8.06(\mathrm{~m}, 5 \mathrm{H}, \mathrm{Ar}-\mathrm{H}), 8.08(\mathrm{~d}, 2 \mathrm{H}, J=$ $8.8 \mathrm{~Hz}, \mathrm{Ar}-\mathrm{H}), 8.10(\mathrm{~d}, 2 \mathrm{H}, J=6.6 \mathrm{~Hz}, \operatorname{Ar}-\mathrm{H}), 8.14(\mathrm{~d}, 2 \mathrm{H}, J=$ $7.6 \mathrm{~Hz}, \mathrm{Ar}-\mathrm{H}), 8.18$ (d, $2 \mathrm{H}, J=4.4 \mathrm{~Hz}, \mathrm{Ar}-\mathrm{H}), 8.20-8.22(\mathrm{~m}$, $3 \mathrm{H}, \mathrm{Ar}-\mathrm{H}$ ); ${ }^{13} \mathrm{C}$ NMR (DMSO-d6) $\delta$; $52.5\left(1 \mathrm{C}, \mathrm{OCH}_{3}\right), 128.2$ (2C), 128.8 (2C), 130.6, 132.5 (phenyl), 42.0, 85.6, 155.6 (3C, isoxazole), 125.8, 127.4 (2C), 132.3 (2C), 139.4 (Ar-C), 125.2 (2C), 127.5 (2C), 131.8, 137.2 (Ar-C), 118.2, 120.5, 131.4, 133.4, 136.8, 152.5, 157.3 (quinazoline), 162.5 (1C, $\mathrm{C}=\mathrm{O}) ; \%$ Anal. Cal. for $\mathrm{C}_{30} \mathrm{H}_{22} \mathrm{ClN}_{3} \mathrm{O}_{3} ; \mathrm{C}, 70.93 ; \mathrm{N}, 8.27 ; \mathrm{H}, 4.37$. Found; $\mathrm{C}$, 70.92; N, 8.25; H, 4.35; Ms (m/z): $507.96(\mathrm{M}+1)$.

(9) 7-Methyl-2-phenyl-3-(4-(3-phenyl-4,5-dihydroisoxazol-5yl)phenyl)quinazolin-4(3H)-one (9). Yield: 55\%; m.p. 133$135^{\circ} \mathrm{C}$; TLC solvent (B: A, 9:1); $R_{f} 0.80 ; \mathrm{IR}\left(\mathrm{KBr}, \mathrm{cm}^{-1}\right): 3012$ (Ar-CH), $1690(\mathrm{CO}), 1610(\mathrm{C}=\mathrm{N}), 1588,1420(\mathrm{C}=\mathrm{C}), 1232(\mathrm{C}-$ $\mathrm{O}-\mathrm{N}), 1100(\mathrm{C}-\mathrm{N}) ;{ }^{1} \mathrm{H}$ NMR (DMSO-d6) $\delta(\mathrm{ppm}) ; 2.30$ (s, $\left.3 \mathrm{H}, \mathrm{CH}_{3}\right), 3.97\left(\mathrm{~d}, 2 \mathrm{H}, J=4.5 \mathrm{~Hz}, \mathrm{CH}_{2 \mathrm{isox}}\right), 5.86(\mathrm{~d}, 1 \mathrm{H}, J=$ $7.8 \mathrm{~Hz}, \mathrm{CH}_{\text {isox. }}$ ), 7.94-8.00 (m, 5H, Ar-H), 8.02-8.04 (m, $3 \mathrm{H}$, Ar-H), 8.07 (t, $1 \mathrm{H}, J=2.8,6.0 \mathrm{~Hz}, \operatorname{Ar}-\mathrm{H}), 8.10(\mathrm{~d}, 2 \mathrm{H}, J=$
$6.9 \mathrm{~Hz}, \mathrm{Ar}-\mathrm{H}), 8.13(\mathrm{~d}, 2 \mathrm{H}, J=7.7 \mathrm{~Hz}, \mathrm{Ar}-\mathrm{H}), 8.17-8.20(\mathrm{~m}$, $4 \mathrm{H}, \mathrm{Ar}-\mathrm{H}) ;{ }^{13} \mathrm{C}$ NMR (DMSO-d6) $\delta ; 21.5\left(1 \mathrm{C}, \mathrm{CH}_{3}\right), 128.2$ (2C), 128.8 (2C), 131.5, 132.5 (phenyl), 42.0, 83.2, 153.5 (3C, isoxazole), 124.3, 128.8 (2C), 131.2 (2C), 132.3 (Ar-C), 125.8 (2C), 128.2 (2C), 130.8, 138.0 (Ar-C), 118.5, 120.6, 132.4, 133.8, 142.0, 151.2, 156.7 (quinazoline), 162.4 (1C, $\mathrm{C}=\mathrm{O}) ; \%$ Anal. Cal. for $\mathrm{C}_{30} \mathrm{H}_{23} \mathrm{~N}_{3} \mathrm{O}_{2} ; \mathrm{C}, 78.75 ; \mathrm{N}, 9.18 ; \mathrm{H}, 5.07$. Found; C, 78.76; N, 9.16; H, 5.05; Ms $(m / z): 457.52\left(\mathrm{M}^{+}\right)$.

(10) 2-(2-Chlorophenyl)-7-methyl-3-(4-(3-phenyl-4,5-dihydroisoxazol-5-yl)phenyl)quinazolin-4(3H)-one (10). Yield: 60\%; m.p. $154-156^{\circ} \mathrm{C}$; TLC solvent (T:E:F, $\left.5: 4: 1\right) ; R_{f} 0.71$; IR $\left(\mathrm{KBr}, \mathrm{cm}^{-1}\right): 3002(\mathrm{Ar}-\mathrm{CH}), 1710(\mathrm{CO}), 1624(\mathrm{C}=\mathrm{N}), 1608$, $1488(\mathrm{C}=\mathrm{C}), 1220(\mathrm{C}-\mathrm{O}-\mathrm{N}), 1105(\mathrm{C}-\mathrm{N}), 710(\mathrm{C}-\mathrm{Cl}) ;{ }^{1} \mathrm{H}$ NMR (DMSO- $\left.d_{6}\right) \delta: 2.22\left(\mathrm{~s}, 3 \mathrm{H}, \mathrm{CH}_{3}\right), 3.87\left(\mathrm{~d}, 2 \mathrm{H}, \mathrm{CH}_{2 \text { isox. }}\right)$, 5.81 (dd, $1 \mathrm{H}, \mathrm{CH}_{\text {isox. }}$ ), 8.02-7.98 (m, 5H, Ar-H), 8.04-8.02 (d, 2H, Ar-H), 8.07-8.03 (d, 2H, Ar-H), 8.10-8.08 (d, $2 \mathrm{H}$, Ar-H), 8.16-8.13 (d, 2H, Ar-H), 8.22-8.19 (m, 3H, Ar-H); ${ }^{13} \mathrm{C}$ NMR (DMSO- $\left.d_{6}\right) \delta ; 13.46\left(1 \mathrm{C}, \mathrm{CH}_{3}\right), 106.16,108.65,110.54$, $114.20,122.62,121.13$ (6C, phenyl), 63.44, 150.12, 154.12 (3C, isoxazole), 115.82, 122.13, 134.12, 134.84, 138.12, 154.25 (6C, $\left.\mathrm{Ar}_{2}-\mathrm{C}\right), 108.16,115.44,118.65,126.52,127.06,133.24$ (6C, $\left.\mathrm{Ar}_{1}-\mathrm{C}\right), 108.65,110.58,115.17,126.52,150.53,147.62$, 160.62 (7C, quinazoline), $171.66(1 \mathrm{C}, \mathrm{C}=\mathrm{O}) ; \%$ Anal. Cal. for $\mathrm{C}_{30} \mathrm{H}_{22} \mathrm{ClN}_{3} \mathrm{O}_{2} ; \mathrm{C}, 73.24 ; \mathrm{N}, 8.54 ; \mathrm{H}, 4.51$. Found; C, 73.22; $\mathrm{N}, 8.52 ; \mathrm{H}, 4.50 ; \mathrm{Ms}(\mathrm{m} / \mathrm{z}): 492.96(\mathrm{M}+1)$.

(11) 7-Methyl-3-(4-(3-phenyl-4,5-dihydroisoxazol-5-yl)phenyl) -2-p-tolylquinazolin-4(3H)-one (11). Yield: 66\%; m.p. 178$180^{\circ} \mathrm{C}$; TLC solvent (T:E: F, $\left.5: 4: 1\right) ; R_{f} 0.58$; IR $\left(\mathrm{KBr}, \mathrm{cm}^{-1}\right)$ : 3012 (Ar-CH), 1678 (CO), 1612 (C=N), 1572, 1453 (C=C), 1218 (C-O-N), $1112(\mathrm{C}-\mathrm{N}) ;{ }^{1} \mathrm{H}$ NMR (DMSO-d6) $\delta(\mathrm{ppm}) ; 2.21$ (s, $\left.3 \mathrm{H}, \mathrm{CH}_{3}\right), 2.36\left(\mathrm{~s}, 3 \mathrm{H}, \mathrm{CH}_{3}\right), 3.80\left(\mathrm{~d}, 2 \mathrm{H}, J=2.1 \mathrm{~Hz}, \mathrm{CH}_{2 \text { isox. }}\right)$, $5.74\left(\mathrm{dd}, 1 \mathrm{H}, J=4.5,2.4 \mathrm{~Hz}, \mathrm{CH}_{\text {isox. }}\right), 8.04-8.08(\mathrm{~m}, 5 \mathrm{H}, \mathrm{Ar}-$ $\mathrm{H}), 8.10(\mathrm{~d}, 2 \mathrm{H}, J=3.3 \mathrm{~Hz}, \operatorname{Ar}-\mathrm{H}), 8.12(\mathrm{~d}, 2 \mathrm{H}, J=4.5 \mathrm{~Hz}$, Ar-H), 8.15 (d, $2 \mathrm{H}, J=4.5 \mathrm{~Hz}, \mathrm{Ar}-\mathrm{H}), 8.22(\mathrm{~d}, 2 \mathrm{H}, J=7.2 \mathrm{~Hz}$, Ar-H), 8.30-8.32 (m, 3H, Ar-H); ${ }^{13} \mathrm{C}$ NMR (DMSO-d6) $\delta$; $21.6\left(2 \mathrm{C}, \mathrm{CH}_{3}\right), 128.0(2 \mathrm{C}), 128.6(2 \mathrm{C}), 131.2,132.5$ (phenyl), 42.2, 85.5, 153.6 (3C, isoxazole), 124.2, 127.6 (2C), 130.7 (2C), 139.2 (Ar-C), 125.8 (2C), 128.3 (2C), 130.8, 136.2 (Ar-C), 118.8, $120.3,130.6,133.2,143.2,152.4,157.4$ (quinazoline), 160.4 (1C, $\mathrm{C}=\mathrm{O}$ ); \% Anal. Cal. for $\mathrm{C}_{31} \mathrm{H}_{25} \mathrm{~N}_{3} \mathrm{O}_{2} ; \mathrm{C}, 78.96 ; \mathrm{N}, 8.91 ; \mathrm{H}$, 5.34. Found; C, 78.97; N, 8.90; H, 5.31; $\mathrm{Ms}(\mathrm{m} / z): 471.54\left(\mathrm{M}^{+}\right)$.

(12) 2-(4-Methoxyphenyl)-7-methyl-3-(4-(3-phenyl-4,5-dihydroisoxazol-5-yl)phenyl)quinazolin-4(3H)-one (12). Yield: 76\%; m.p. $172-174^{\circ} \mathrm{C}$; TLC solvent (T:E: F, $\left.5: 4: 1\right) ; R_{f} 0.58$; IR $\left(\mathrm{KBr}, \mathrm{cm}^{-1}\right)$ : $3012(\mathrm{Ar}-\mathrm{CH}), 1677(\mathrm{CO}), 1612(\mathrm{C}=\mathrm{N})$, 1596, $1453(\mathrm{C}=\mathrm{C}), 1300(\mathrm{C}-\mathrm{O}), 1226(\mathrm{C}-\mathrm{O}-\mathrm{N}), 1106(\mathrm{C}-\mathrm{N})$; ${ }^{1} \mathrm{H}$ NMR (DMSO-d6) $\delta(\mathrm{ppm}) ; 2.28\left(\mathrm{~s}, 3 \mathrm{H}, \mathrm{CH}_{3}\right), 2.82(\mathrm{~s}$, $\left.3 \mathrm{H}, \mathrm{OCH}_{3}\right), 3.86\left(\mathrm{~d}, 2 \mathrm{H}, J=7.0 \mathrm{~Hz}, \mathrm{CH}_{2 \text { isox. }}\right), 5.89(\mathrm{~m}, 1 \mathrm{H}$, $\mathrm{CH}_{\text {isox }}$ ), $7.96-7.94(\mathrm{~m}, 5 \mathrm{H}, \mathrm{Ar}-\mathrm{H}), 8.08(\mathrm{~d}, 2 \mathrm{H}, J=7.5 \mathrm{~Hz}$, $\mathrm{Ar}-\mathrm{H}), 8.14(\mathrm{~d}, 2 \mathrm{H}, J=7.4 \mathrm{~Hz}, \mathrm{Ar}-\mathrm{H}), 8.18(\mathrm{~d}, 2 \mathrm{H}, J=8.4 \mathrm{~Hz}$, $\mathrm{Ar}-\mathrm{H}), 8.20(\mathrm{~d}, 2 \mathrm{H}, J=6.6 \mathrm{~Hz}, \mathrm{Ar}-\mathrm{H}), 8.21-8.23(\mathrm{~m}, 3 \mathrm{H}$, $\mathrm{Ar}-\mathrm{H}) ;{ }^{13} \mathrm{C}$ NMR (DMSO-d6) $\delta ; 20.4\left(1 \mathrm{C}, \mathrm{CH}_{3}\right), 50.5$ (1C, $\left.\mathrm{OCH}_{3}\right) 128.2(2 \mathrm{C}), 128.9$ (2C), 131.6, 132.5 (phenyl), 42.0, 84.8, 153.5 (3C, isoxazole), 125.2, 128.0 (2C), 131.4 (2C), 138.5 (Ar-C), 125.6 (2C), 128.0 (2C), 132.4, 138.2 (Ar-C), 120.8, 122.6, 130.6, 133.4, 138.0, 151.4, 155.7 (quinazoline), 161.5 (1C, 
$\mathrm{C}=\mathrm{O}$ ); \% Anal. Cal. for $\mathrm{C}_{31} \mathrm{H}_{25} \mathrm{~N}_{3} \mathrm{O}_{3} ; \mathrm{C}, 76.37 ; \mathrm{N}, 8.62 ; \mathrm{H}$, 5.17. Found; C, 76.32; N, 8.60; H, 5.15; Ms $(m / z): 488.54$ $(\mathrm{M}+1)$.

(13) 3-(4-(3-(4-Chlorophenyl)-4, 5-dihydroisoxazol-5-yl) phenyl)-2-phenylquinazolin-4(3H)-one (13). Yield: 70\%; m.p. $145-147^{\circ} \mathrm{C}$; TLC solvent (B : A, $\left.9: 1\right) ; R_{f} 0.70$; IR $\left(\mathrm{KBr}, \mathrm{cm}^{-1}\right)$ : 3012 (Ar-CH), 1684 (CO), $1618(\mathrm{C}=\mathrm{N}), 1596,1473(\mathrm{C}=\mathrm{C})$, $1222(\mathrm{C}-\mathrm{O}-\mathrm{N}), 1102(\mathrm{C}-\mathrm{N}), 712(\mathrm{C}-\mathrm{Cl}) ;{ }^{1} \mathrm{H}$ NMR (DMSOd6) $\delta(\mathrm{ppm}) ; 3.55\left(\mathrm{~d}, 2 \mathrm{H}, J=7.5 \mathrm{~Hz}, \mathrm{CH}_{2 \text { isox. }}\right), 5.92(\mathrm{~m}, 1 \mathrm{H}$, $\mathrm{CH}_{\text {isox. }}$ ), 8.06-8.08 (m, 4H, Ar-H), 8.07-8.09 (m, 4H, Ar-H), $8.10(\mathrm{~d}, 2 \mathrm{H}, J=3.3 \mathrm{~Hz}, \mathrm{Ar}-\mathrm{H}), 8.12(\mathrm{~d}, 2 \mathrm{H}, J=6.6 \mathrm{~Hz}, \mathrm{Ar}-$ $\mathrm{H}), 8.18-8.20$ (m, 5H, Ar-H); ${ }^{13} \mathrm{C}$ NMR (DMSO-d6) $\delta ; 128.0$ (2C), 128.2, 129.2 (2C), 137.2 (phenyl), 42.3, 85.2, 155.6 (3C, isoxazole), 127.2, 128.4 (2C), 128.8 (2C), 130.2 (Ar-C), 125.8 (2C), 128.2 (2C), 130.2, 138.0 (Ar-C), 120.4, 123.5, 130.3, 135.3, 138.0, 148.6, 155.7 (quinazoline), 160.5 (1C, C=O); \% Anal. Cal. for $\mathrm{C}_{29} \mathrm{H}_{20} \mathrm{ClN}_{3} \mathrm{O}_{2} ; \mathrm{C}, 72.88 ; \mathrm{N}, 8.79 ; \mathrm{H}, 4.22$. Found; C, 72.87; $\mathrm{N}, 8.75 ; \mathrm{H}, 4.20 ; \mathrm{Ms}(\mathrm{m} / z)$ : $478.94(\mathrm{M}+1)$.

(14) 2-(2-Chlorophenyl)-3-(4-(3-(4-chlorophenyl)-4,5dihydroisoxazol-5-yl)phenyl)quinazolin-4(3H)-one (14). Yield: 76\%; m.p. $150-152^{\circ} \mathrm{C}$; TLC solvent (T: E : F, $\left.5: 4: 1\right) ; R_{f}$ 0.66; IR (KBr, cm $\left.{ }^{-1}\right)$ : 3033 (Ar-CH), $1698(\mathrm{CO}), 1622(\mathrm{C}=\mathrm{N})$, 1590, 1473 (C=C), $1227(\mathrm{C}-\mathrm{O}-\mathrm{N}), 1132(\mathrm{C}-\mathrm{N}), 752(\mathrm{C}-\mathrm{Cl}) ;{ }^{1} \mathrm{H}$ NMR (DMSO-d6) $\delta$ (ppm); $3.74\left(\mathrm{~d}, 2 \mathrm{H}, J=7.8 \mathrm{~Hz}, \mathrm{CH}_{2 \text { isox. }}\right)$, $5.92\left(\mathrm{~d}, 1 \mathrm{H}, J=2.8 \mathrm{~Hz}, \mathrm{CH}_{\text {isox. }}\right), 8.03-8.05$ (m, 4H, Ar-H), 8.06-8.08 (m, 4H, Ar-H), 8.12 (d, 2H, J = 8.2 Hz, Ar-H), 8.13 $(\mathrm{d}, 2 \mathrm{H}, J=6.8 \mathrm{~Hz}, \mathrm{Ar}-\mathrm{H}), 8.38-8.40(\mathrm{~m}, 4 \mathrm{H}, \mathrm{Ar}-\mathrm{H}) ;{ }^{13} \mathrm{C} \mathrm{NMR}$ (DMSO-d6) $\delta$; 128.2 (2C), 129.8 (2C), 130.8, 137.4 (phenyl), 43.3, 84.6, 156.6 (3C, isoxazole), 126.3, 128.2 (2C), 128.8 (2C), 138.0 (Ar-C), 122.2, 126.8, 130.2, 131.6, 132.0, 132.6 (Ar-C), $120.8,124.6,132.6,133.8,137.4,148.4,155.7$ (quinazoline), 160.5 (1C, $\mathrm{C}=\mathrm{O})$; \% Anal. Cal. for $\mathrm{C}_{29} \mathrm{H}_{19} \mathrm{Cl}_{2} \mathrm{~N}_{3} \mathrm{O}_{2} ; \mathrm{C}, 67.98$; $\mathrm{N}, 8.20 ; \mathrm{H}, 3.74$. Found; C, 67.96; N, 8.18; H, 3.73; Ms $(\mathrm{m} / z)$ : $515(\mathrm{M}+2)$.

(15) 3-(4-(3-(4-Chlorophenyl)-4,5-dihydroisoxazol-5-yl)phenyl)-2-p-tolylquinazolin-4(3H)-one (15). Yield: 77\%; m.p. $145-147^{\circ} \mathrm{C}$; TLC solvent ( $\left.\mathrm{T}: \mathrm{E}: \mathrm{F}, 5: 4: 1\right) ; R_{f}$ 0.67; IR (KBr, $\left.\mathrm{cm}^{-1}\right): 3038$ (Ar-CH), $1710(\mathrm{CO}), 1605(\mathrm{C}=\mathrm{N}), 1590,1453$ $(\mathrm{C}=\mathrm{C}), 1226(\mathrm{C}-\mathrm{O}-\mathrm{N}), 1117(\mathrm{C}-\mathrm{N}), 733(\mathrm{C}-\mathrm{Cl}) ;{ }^{1} \mathrm{H}$ NMR $(\mathrm{DMSO}-\mathrm{d} 6) \delta(\mathrm{ppm}) ; 2.21\left(\mathrm{~s}, 3 \mathrm{H}, \mathrm{CH}_{3}\right), 3.68(\mathrm{~d}, 2 \mathrm{H}, J=$ $\left.9.2 \mathrm{~Hz}, \mathrm{CH}_{\text {2isox. }}\right), 5.86\left(\mathrm{~m}, 1 \mathrm{H}, \mathrm{CH}_{\text {isox. }}\right), 8.06-8.08(\mathrm{~m}, 4 \mathrm{H}$, Ar-H), 8.12-8.14 (m, 4H, Ar-H), 8.18 (d, $2 \mathrm{H}, J=8.2 \mathrm{~Hz}$, Ar-H), 8.19 (d, 2H, J = 7.9 Hz, Ar-H), 8.42-8.44 (m, 4H, Ar-H); ${ }^{13} \mathrm{C}$ NMR (DMSO-d6) $\delta ; 21.8\left(1 \mathrm{C}, \mathrm{CH}_{3}\right), 128.2$ (2C), 128.8 (2C), 131.2, 137.9 (phenyl), 42.2, 83.8, 155.6 (3C, isoxazole), 125.6, 129.2 (2C), 130.5 (2C), 139.2 (Ar-C), 125.8 (2C), 128.5 (2C), 130.8, 138.2 (Ar-C), 120.8, 122.5, 130.3, 133.6, 134.4, 147.4, 157.7 (quinazoline), 162.4 (1C, $\mathrm{C}=\mathrm{O})$; \% Anal. Cal. for $\mathrm{C}_{30} \mathrm{H}_{22} \mathrm{ClN}_{3} \mathrm{O}_{2} ; \mathrm{C}, 73.24 ; \mathrm{N}, 8.24 ; \mathrm{H}, 4.51$. Found; $\mathrm{C}$, 73.22; N, 8.22; H, 4.52; Ms $(m / z)$ : $492.96(\mathrm{M}+1)$.

(16) 3-(4-(3-(4-Chlorophenyl)-4,5-dihydroisoxazol-5-yl) phenyl)-2-(4-methoxyphenyl) quinazolin-4(3H)-one (16). Yield: 66\%; m.p. $164-166^{\circ} \mathrm{C}$; TLC solvent (T:E : F, $5: 4: 1$ ); $R_{f} 0.68$; IR $\left(\mathrm{KBr}, \mathrm{cm}^{-1}\right): 3010$ (Ar-CH), 1696 (CO), $1622(\mathrm{C}=\mathrm{N}), 1598$,
$1466(\mathrm{C}=\mathrm{C}), 1285(\mathrm{C}-\mathrm{O}), 1217(\mathrm{C}-\mathrm{O}-\mathrm{N}), 1120(\mathrm{C}-\mathrm{N}), 717(\mathrm{C}-$ $\mathrm{Cl}) ;{ }^{1} \mathrm{H}$ NMR (DMSO-d6) $\delta$ (ppm); $2.78\left(\mathrm{~s}, 3 \mathrm{H}, \mathrm{OCH}_{3}\right), 3.96$ $\left(\mathrm{d}, 2 \mathrm{H}, J=7.6 \mathrm{~Hz}, \mathrm{CH}_{2 \text { isox. }}\right), 5.92\left(\mathrm{~m}, 1 \mathrm{H}, \mathrm{CH}_{\text {isox. }}\right), 8.04-8.02$ (m, 4H, Ar-H), 8.04-8.06 (m, 4H, Ar-H), 8.08 (d, 2H, J = $4.8 \mathrm{~Hz}, \mathrm{Ar}-\mathrm{H}), 8.10$ (d, $2 \mathrm{H}, J=9.2 \mathrm{~Hz}, \mathrm{Ar}-\mathrm{H}), 8.18-8.20$ (m, $4 \mathrm{H}, \mathrm{Ar}-\mathrm{H}) ;{ }^{13} \mathrm{C}$ NMR (DMSO-d6) $\delta$; $52.6\left(1 \mathrm{C}, \mathrm{OCH}_{3}\right), 128.6$ (2C), 129.2, 130.5 (2C), 137.9 (phenyl), 40.6, 84.3, 156.2 (3C, isoxazole), 115.6 (2C), 121.6, 130.8 (2C), 162.8 (Ar-C), 124.2 (2C), 127.9 (2C), 130.2, 137.8 (Ar-C), 120.2, 126.4, 128.6, 129.2, 133.5, 147.8, 156.2 (quinazoline), 160.2 (1C, $\mathrm{C}=\mathrm{O})$; \% Anal. Cal. for $\mathrm{C}_{30} \mathrm{H}_{22} \mathrm{ClN}_{3} \mathrm{O}_{3} ; \mathrm{C}, 70.93 ; \mathrm{N}, 8.27 ; \mathrm{H}, 4.37$. Found; C, 70.91; $\mathrm{N}, 8.25 ; \mathrm{H}, 4.35$; Ms $(\mathrm{m} / z)$ : $508.96(\mathrm{M}+1)$.

(17) 3-(4-(3-(4-Methoxyphenyl)-4,5-dihydroisoxazol-5-yl)phenyl)-2-phenylquinazolin-4(3H)-one (17). Yield: 60\%; m.p. 190-192 ${ }^{\circ} \mathrm{C}$; TLC solvent (T:E : F, $\left.5: 4: 1\right) ; R_{f} 0.70$; IR (KBr, $\left.\mathrm{cm}^{-1}\right)$ : 3018 (Ar-CH), $1713(\mathrm{CO}), 1634(\mathrm{C}=\mathrm{N}), 1586,1453$ $(\mathrm{C}=\mathrm{C}), 1296(\mathrm{C}-\mathrm{O}), 1234(\mathrm{C}-\mathrm{O}-\mathrm{N}), 1110(\mathrm{C}-\mathrm{N}) ;{ }^{1} \mathrm{H}$ NMR (DMSO-d6) $\delta$ (ppm); $2.74\left(\mathrm{~s}, 3 \mathrm{H}, \mathrm{OCH}_{3}\right), 3.68(\mathrm{~d}, 2 \mathrm{H}, J=$ $2.9 \mathrm{~Hz}, \mathrm{CH}_{\text {2isox. }}$ ), 5.92 (d, $1 \mathrm{H}, J=8.4 \mathrm{~Hz}, \mathrm{CH}_{\text {isox. }}$ ), 8.00-8.02 (m, 4H, Ar-H), 8.04-8.06 (m, 4H, Ar-H), 8.15 (d, 2H, J = $8.3 \mathrm{~Hz}, \mathrm{Ar}-\mathrm{H}), 8.16$ (d, 2H, $J=7.4 \mathrm{~Hz}, \mathrm{Ar}-\mathrm{H}), 8.38-8.40$ (m, $5 \mathrm{H}, \mathrm{Ar}-\mathrm{H}) ;{ }^{13} \mathrm{C}$ NMR (DMSO-d6) $\delta$; $54.6\left(1 \mathrm{C}, \mathrm{OCH}_{3}\right), 115.8$ (2C), 122.5, 127.3 (2C), 160.6 (phenyl), 40.8, 84.2, 156.4 (3C, isoxazole), 127.2 (2C), 128.2 (2C), 128.8, 130.6 (Ar-C), 126.8 (2C), 127.8 (2C), 131.4, 137.5 (Ar-C), 120.4, 126.5, 127.3, 129.4, 134.8, 148.0, 155.4 (quinazoline), 161.2 (1C, C=O); \% Anal. Cal. for $\mathrm{C}_{30} \mathrm{H}_{23} \mathrm{~N}_{3} \mathrm{O}_{3} ; \mathrm{C}, 76.09 ; \mathrm{N}, 8.87 ; \mathrm{H}, 4.90$. Found; C, 76.07; $\mathrm{N}, 8.85 ; \mathrm{H}, 4.92 ; \mathrm{Ms}(\mathrm{m} / z): 475.52(\mathrm{M}+2)$.

(18) 2-(2-Chlorophenyl)-3-(4-(3-(4-methoxyphenyl)-4,5-dihydroisoxazol-5-yl)phenyl)quinazolin-4(3H)-one (18). Yield: 70\%; m.p. 165-167 C; TLC solvent (B:A, 9:1); $R_{f}$ 0.63; IR $\left(\mathrm{KBr}, \mathrm{cm}^{-1}\right): 3018$ (Ar-CH), $1713(\mathrm{CO}), 1634(\mathrm{C}=\mathrm{N}), 1586$, $1453(\mathrm{C}=\mathrm{C}), 1308(\mathrm{C}-\mathrm{O}), 1234(\mathrm{C}-\mathrm{O}-\mathrm{N}), 1110(\mathrm{C}-\mathrm{N}) ;{ }^{1} \mathrm{H}$ NMR (DMSO-d6) $\delta$ (ppm); $2.68\left(\mathrm{~s}, 3 \mathrm{H}, \mathrm{OCH}_{3}\right), 3.88$ (d, $\left.2 \mathrm{H}, J=6.4 \mathrm{~Hz}, \mathrm{CH}_{2 \text { isox. }}\right), 5.80\left(\mathrm{~m}, 1 \mathrm{H}, \mathrm{CH}_{\text {isox. }}\right), 8.06-8.08$ (m, 4H, Ar-H), 8.10-8.12 (m, 4H, Ar-H), 8.15 (d, 2H, $J=$ $7.8 \mathrm{~Hz}, \mathrm{Ar}-\mathrm{H}), 8.30$ (d, 2H, $J=8.0 \mathrm{~Hz}, \mathrm{Ar}-\mathrm{H}), 8.38-8.40$ (m, $4 \mathrm{H}, \mathrm{Ar}-\mathrm{H}) ;{ }^{13} \mathrm{C}$ NMR (DMSO-d6) $\delta ; 55.2\left(1 \mathrm{C}, \mathrm{OCH}_{3}\right), 114.5$ (2C), 123.5, 128.8 (2C), 161.2 (phenyl), 42.4, 83.8, 157.4 (3C, isoxazole), 123.6, 126.8, 129.2, 130.4, 131.8, 132.5 (Ar-C), 125.6 (2C), 128.2 (2C), 130.4, 138.5 (Ar-C), 120.4, 126.2, 126.8, 127.9, 133.8, 147.5, 154.5 (quinazoline), 160.5 (1C, $\mathrm{C}=\mathrm{O}$ ); \% Anal. Cal. for $\mathrm{C}_{30} \mathrm{H}_{22} \mathrm{ClN}_{3} \mathrm{O}_{3}$; C, 70.93; N, 8.27; H, 4.37. Found; C, 70.95; N, 8.25; H, 4.35; Ms $(m / z): 508.96(\mathrm{M}+1)$.

(19) 3-(4-(3-(4-Methoxyphenyl)-4,5-dihydroisoxazol-5-yl) phenyl)-2-p-tolylquinazolin-4(3H)-one (19). Yield: 73\%; m.p. $176-178^{\circ} \mathrm{C}$; TLC solvent (B : A, $\left.9: 1\right) ; R_{f} 0.60$; IR $\left(\mathrm{KBr}, \mathrm{cm}^{-1}\right)$ : 3015 (Ar-CH), 1697 (CO), 1618 (C=N), 1588, 1468 (C=C), 1280 (C-O), $1230(\mathrm{C}-\mathrm{O}-\mathrm{N}), 1154(\mathrm{C}-\mathrm{N}) ;{ }^{1} \mathrm{H}$ NMR (DMSO-d6) $\delta$ (ppm); 2.03 (s, 3H, $\left.\mathrm{CH}_{3}\right), 2.56\left(\mathrm{~s}, 3 \mathrm{H}, \mathrm{OCH}_{3}\right), 3.80(\mathrm{~d}, 2 \mathrm{H}, J=$ $\left.8.8 \mathrm{~Hz}, \mathrm{CH}_{2 \text { isox. }}\right), 5.88\left(\mathrm{~d}, 1 \mathrm{H}, J=9.2 \mathrm{~Hz}, \mathrm{CH}_{\text {isox. }}\right), 8.04(\mathrm{~d}, 2 \mathrm{H}$, $J=9.2 \mathrm{~Hz}, \mathrm{Ar}-\mathrm{H}), 8.06$ (d, $2 \mathrm{H}, J=6.4 \mathrm{~Hz}, \mathrm{Ar}-\mathrm{H}), 8.10$ (d, $2 \mathrm{H}$, $J=3.6 \mathrm{~Hz}, \mathrm{Ar}-\mathrm{H}), 8.12(\mathrm{t}, 2 \mathrm{H}, J=8.0,7.4 \mathrm{~Hz}, \mathrm{Ar}-\mathrm{H}), 8.14(\mathrm{~d}$, $2 \mathrm{H}, J=4.4 \mathrm{~Hz}, \mathrm{Ar}-\mathrm{H}), 8.16$ (d, $2 \mathrm{H}, J=8.4$, Ar-H), 8.15-8.17 (m, 4H, Ar-H); ${ }^{13} \mathrm{C}$ NMR (DMSO-d6) $\delta ; 20.4\left(1 \mathrm{C}, \mathrm{CH}_{3}\right), 55.3$ 
(1C, $\mathrm{OCH}_{3}$ ), 114.4 (2C), 120.3, 128.8 (2C), 161.5 (phenyl), 42.2, 83.0, 155.6 (3C, isoxazole), 125.2, 129.5 (2C), 130.3 (2C), 140.2 (Ar-C), 125.2 (2C), 128.2 (2C), 130.4, 138.2 (Ar-C), 120.4, $126.2,127.8,130.4,133.8,148.8,156.2$ (quinazoline), 160.8 (1C, $\mathrm{C}=\mathrm{O}$ ); \% Anal. Cal. for $\mathrm{C}_{31} \mathrm{H}_{25} \mathrm{~N}_{3} \mathrm{O}_{3} ; \mathrm{C}, 76.37 ; \mathrm{N}, 8.62 ; \mathrm{H}$, 5.17. Found; C, 76.35; N, 8.60; H, 5.19; $\mathrm{Ms}(\mathrm{m} / z): 487.54\left(\mathrm{M}^{+}\right)$.

(20) 2-(4-Methoxyphenyl)-3-(4-(3-(4-methoxyphenyl)-4,5dihydroisoxazol-5-yl)phenyl) quinazolin-4(3H)-one (20). Yield: $65 \%$; m.p. $162-164^{\circ} \mathrm{C}$; TLC solvent (T: E : F, $\left.5: 4: 1\right) ; R_{f}$ 0.55; IR (KBr, cm $\left.{ }^{-1}\right): 3024$ (Ar-CH), $1688(\mathrm{CO}), 1612(\mathrm{C}=\mathrm{N})$, 1595, $1472(\mathrm{C}=\mathrm{C}), 1298(\mathrm{C}-\mathrm{O}), 1224(\mathrm{C}-\mathrm{O}-\mathrm{N}), 1160(\mathrm{C}-\mathrm{N})$; ${ }^{1} \mathrm{H}$ NMR (DMSO-d6) $\delta(\mathrm{ppm}) ; 2.80$ (s, 3H, $\left.\mathrm{OCH}_{3}\right), 2.86(\mathrm{~s}$, $\left.3 \mathrm{H}, \mathrm{OCH}_{3}\right), 3.84\left(\mathrm{~d}, 2 \mathrm{H}, \mathrm{J}=5.8 \mathrm{~Hz}, \mathrm{CH}_{2 \text { isox. }}\right), 5.92(\mathrm{~m}, 1 \mathrm{H}$, $\mathrm{CH}_{\text {isox. }}$ ), 8.08-8.10 (m, 4H, Ar-H), 8.12-8.14 (m, 4H, Ar-H), 8.15 (d, $2 \mathrm{H}, J=8.0 \mathrm{~Hz}, \mathrm{Ar}-\mathrm{H}), 8.17$ (d, $2 \mathrm{H}, J=9.4 \mathrm{~Hz}, \mathrm{Ar}-\mathrm{H})$, 8.20-8.22 (m, 4H, Ar-H); ${ }^{13} \mathrm{C}$ NMR (DMSO-d6) $\delta$; 54.7(2C, $\mathrm{OCH}_{3}$ ), 116.2 (2C), 122.8, 127.2 (2C), 162.3 (phenyl), 42.5, 84.5, 156.2 (3C, isoxazole), 115.6 (2C), 120.3, 130.3 (2C), 160.8 (Ar-C), 125.8 (2C), 129.5 (2C), 131.5, 137.5 (Ar-C), 120.3, 126.6, $128.3,130.8,132.5,147.3,157.5$ (quinazoline), $162.3(1 \mathrm{C}, \mathrm{C}=\mathrm{O})$; \% Anal. Cal. for $\mathrm{C}_{31} \mathrm{H}_{25} \mathrm{~N}_{3} \mathrm{O}_{4} ; \mathrm{C}, 73.94 ; \mathrm{N}, 8.34 ; \mathrm{H}, 5.00$. Found; C, 73.92; N, 8.32; H, 5.02; Ms $(m / z): 503.54\left(\mathrm{M}^{+}\right)$.

(21) 7-Chloro-3-(4-(3-(4-chlorophenyl)-4,5-dihydroisoxazol-5yl)phenyl)-2-phenylquinazolin-4(3H)-one (21). Yield: $68 \%$; m.p. $180-182^{\circ} \mathrm{C}$; TLC solvent (T:E:F, 5:4:1); $R_{f} 0.54$; IR $\left(\mathrm{KBr}, \mathrm{cm}^{-1}\right): 3032(\mathrm{Ar}-\mathrm{CH}), 1684(\mathrm{CO}), 1608(\mathrm{C}=\mathrm{N}), 1592$, $1426(\mathrm{C}=\mathrm{C}), 1228(\mathrm{C}-\mathrm{O}-\mathrm{N}), 1124(\mathrm{C}-\mathrm{N}), 712(\mathrm{C}-\mathrm{Cl}) ;{ }^{1} \mathrm{H}$ NMR (DMSO-d6) $\delta$ (ppm); $3.93\left(\mathrm{~d}, 2 \mathrm{H}, J=7.8 \mathrm{~Hz}, \mathrm{CH}_{2 \text { isox. }}\right), 5.95$ (d, $1 \mathrm{H}, J=8.3 \mathrm{~Hz}, \mathrm{CH}_{\text {isox. }}$ ), 8.10-8.12 (m, 4H, Ar-H), 8.14$8.16(\mathrm{~m}, 3 \mathrm{H}, \mathrm{Ar}-\mathrm{H}), 8.20$ (d, $2 \mathrm{H}, J=8.4 \mathrm{~Hz}, \mathrm{Ar}-\mathrm{H}), 8.22$ (d, $2 \mathrm{H}, J=7.2 \mathrm{~Hz}, \mathrm{Ar}-\mathrm{H}), 8.28-8.32(\mathrm{~m}, 5 \mathrm{H}, \mathrm{Ar}-\mathrm{H}) ;{ }^{13} \mathrm{C} \mathrm{NMR}$ (DMSO-d6) $\delta$; 128.2 (2C), 128.8, 129.2 (2C), 137.2 (phenyl), 41.5, 85.0, 156.2 (3C, isoxazole), 128.2 (2C), 128.6, 129.5 (2C), 130.7 (Ar-C), 125.5 (2C), 127.8 (2C), 131.4, 137.6 (Ar-C), 118.4, $125.2,126.8,132.4,137.5,152.2,157.5$ (quinazoline), 160.8 (1C, $\mathrm{C}=\mathrm{O}) ; \%$ Anal. Cal. for $\mathrm{C}_{29} \mathrm{H}_{19} \mathrm{Cl}_{2} \mathrm{~N}_{3} \mathrm{O}_{2} ; \mathrm{C}, 67.98 ; \mathrm{N}, 8.20 ; \mathrm{H}$, 3.74. Found; C, 67.96; N, 8.17; H, 3.72; Ms $(m / z)$ : $513.38(\mathrm{M}+1)$.

(22) 7-Chloro-2-(2-chlorophenyl)-3-(4-(3-(4-chlorophenyl)4,5-dihydroisoxazol-5-yl)phenyl)quinazolin-4(3H)-one (22). Yield: 75\%; m.p. $157-159^{\circ} \mathrm{C}$; TLC solvent (T: E : F, $\left.5: 4: 1\right) ; R_{f}$ 0.67; IR (KBr, cm $\left.{ }^{-1}\right): 3042(\mathrm{Ar}-\mathrm{CH}), 1688(\mathrm{CO}), 1628(\mathrm{C}=\mathrm{N})$, 1587, 1420 (C=C), 1225 (C-O-N), $1113(\mathrm{C}-\mathrm{N}), 734(\mathrm{C}-\mathrm{Cl})$; ${ }^{1} \mathrm{H}$ NMR (DMSO-d6) $\delta(\mathrm{ppm}) ; 3.84(\mathrm{~d}, 2 \mathrm{H}, J=5.4 \mathrm{~Hz}$, $\left.\mathrm{CH}_{2 \text { isox. }}\right), 5.90\left(\mathrm{~m}, 1 \mathrm{H}, \mathrm{CH}_{\text {isox. }}\right), 8.10-8.12(\mathrm{~m}, 4 \mathrm{H}, \mathrm{Ar}-\mathrm{H})$, 8.12-8.14 (m, 3H, Ar-H), 8.16 (d, $2 \mathrm{H}, J=7.8 \mathrm{~Hz}$, Ar-H), 8.18 (d, $2 \mathrm{H}, J=9.4 \mathrm{~Hz}, \mathrm{Ar}-\mathrm{H}), 8.18-8.20$ (m, 4H, Ar-H); ${ }^{13} \mathrm{C} \mathrm{NMR}$ (DMSO-d6) $\delta$; 118.4 (2C), 122.6, 128.2 (2C), 136.2 (phenyl), 42.5, 84.0, 157.6 (3C, isoxazole), 123.5, 126.5, 130.3, 131.2, 132.3, 132.8 (Ar-C), 124.8 (2C), 128.6 (2C), 130.2, 137.5 (Ar-C), 118.4, $122.2,125.6,129.4,137.5,150.8,157.4$ (quinazoline), $162.4(1 \mathrm{C}$, $\mathrm{C}=\mathrm{O}$ ); \% Anal. Cal. for $\mathrm{C}_{29} \mathrm{H}_{18} \mathrm{Cl}_{3} \mathrm{~N}_{3} \mathrm{O}_{2} ; \mathrm{C}, 63.70 ; \mathrm{N}, 7.68$; $\mathrm{H}, 3.32$. Found; C, 63.72; N, 7.66; H, 3.30; Ms $(\mathrm{m} / z): 547.83$ $(\mathrm{M}+1)$.
(23) 7-Chloro-3-(4-(3-(4-chlorophenyl)-4,5-dihydroisoxazol5-yl)phenyl)-2-p-tolylquinazolin-4(3H)-one (23). Yield: 74\%; m.p. $181-183^{\circ} \mathrm{C}$; TLC solvent (T:E:F, $5: 4: 1$ ); $R_{f} 0.70$; IR $\left(\mathrm{KBr}, \mathrm{cm}^{-1}\right): 3038$ (Ar-CH), $1694(\mathrm{CO}), 1622(\mathrm{C}=\mathrm{N}), 1595$, 1420 (C=C), 1305 (C-O), $1216(\mathrm{C}-\mathrm{O}-\mathrm{N}), 1114(\mathrm{C}-\mathrm{N}), 710$ $(\mathrm{C}-\mathrm{Cl}) ;{ }^{1} \mathrm{H}$ NMR (DMSO-d6) $\delta$ (ppm); 2.23 (s, 3H, $\mathrm{CH}_{3}$ ), $3.76\left(\mathrm{~d}, 2 \mathrm{H}, J=7.2 \mathrm{~Hz}, \mathrm{CH}_{2 \text { isox. }}\right), 5.86(\mathrm{~d}, 1 \mathrm{H}, J=10.4 \mathrm{~Hz}$, $\mathrm{CH}_{\text {isox. }}$ ), 7.96-7.98 (m, 4H, Ar-H), 8.02-8.04 (m, 4H, Ar-H), 8.06-8.08 (m, 4H, Ar-H), 8.16 (d, 2H, $J=8.8 \mathrm{~Hz}, \mathrm{Ar}-\mathrm{H})$, $8.20(\mathrm{~d}, 2 \mathrm{H}, J=8.0 \mathrm{~Hz}, \mathrm{Ar}-\mathrm{H}) ;{ }^{13} \mathrm{C}$ NMR (DMSO-d6) $\delta ; 22.5$ (1C, $\mathrm{CH}_{3}$ ), 128.2 (2C), 128.6, 128.8 (2C), 135.8 (phenyl), 43.0, 83.5, 156.2 (3C, isoxazole), 125.8, 129.0 (2C), 130.8 (2C), 138.8 (Ar-C), 125.8 (2C), 127.8 (2C), 131.2, 138.0 (Ar-C), 118.8, 122.7, $127.5,130.6,137.4,152.5,156.4$ (quinazoline), 162.3 (1C, $\mathrm{C}=\mathrm{O})$; \% Anal. Cal. for $\mathrm{C}_{30} \mathrm{H}_{21} \mathrm{Cl}_{2} \mathrm{~N}_{3} \mathrm{O}_{2} ; \mathrm{C}, 68.45 ; \mathrm{N}, 7.98 ; \mathrm{H}, 4.02$. Found; C, 68.47; N, 7.96; H, 4.00; Ms $(\mathrm{m} / z)$ : $528.41(\mathrm{M}+2)$.

(24) 7-Chloro-3-(4-(3-(4-chlorophenyl)-4,5-dihydroisoxazol5-yl)phenyl)-2-(4-methoxyphenyl)quinazolin-4(3H)-one (24). Yield: $80 \%$; m.p. $186-188^{\circ} \mathrm{C}$; TLC solvent (B : A, 9:1); $R_{f} 0.69$; IR $\left(\mathrm{KBr}, \mathrm{cm}^{-1}\right)$ : 3036 (Ar-CH), $1680(\mathrm{CO}), 1622(\mathrm{C}=\mathrm{N}), 1596$, 1420 (C=C), 1300 (C-O), $1233(\mathrm{C}-\mathrm{O}-\mathrm{N}), 1130(\mathrm{C}-\mathrm{N}), 735$ $(\mathrm{C}-\mathrm{Cl}) ;{ }^{1} \mathrm{H}$ NMR (DMSO-d6) $\delta$ (ppm); 2.65 (s, 3H, $\mathrm{OCH}_{3}$ ), $3.84\left(\mathrm{~d}, 2 \mathrm{H}, J=8.0 \mathrm{~Hz}, \mathrm{CH}_{2 \text { isox. }}\right), 6.01\left(\mathrm{~m}, 1 \mathrm{H}, \mathrm{CH}_{\text {isox. }}\right)$, 7.98-8.00 (m, 4H, Ar-H), 8.04-8.06 (m, 3H, Ar-H), 8.08-8.10 (m, 4H, Ar-H), 8.14 (d, 2H, J = 8.0 Hz, Ar-H), 8.18 (d, 2H, J $=4.8 \mathrm{~Hz}, \mathrm{Ar}-\mathrm{H}) ;{ }^{13} \mathrm{C}$ NMR (DMSO-d6) $\delta ; 52.4\left(1 \mathrm{C}, \mathrm{OCH}_{3}\right)$, 128.3 (2C), 128.5, 128.9 (2C), 136.4 (phenyl), 43.8, 82.6, 158.6 (3C, isoxazole), 115.5 (2C), 120.8, 131.5 (2C), 162.4 (Ar-C), 125.2 (2C), 128.5 (2C), 132.6, 138.5 (Ar-C), 118.2, 120.5, 128.3, $131.4,137.8,151.4,157.3$ (quinazoline), $160.2(1 \mathrm{C}, \mathrm{C}=\mathrm{O}) ; \%$ Anal. Cal. for $\mathrm{C}_{30} \mathrm{H}_{21} \mathrm{Cl}_{2} \mathrm{~N}_{3} \mathrm{O}_{3} ; \mathrm{C}, 66.43 ; \mathrm{N}, 7.75 ; \mathrm{H}, 3.90$. Found; C, 66.41; N, 7.73; H, 3.88; Ms (m/z): $544.11(\mathrm{M}+2)$.

(25) 3-(4-(3-(4-Chlorophenyl)-4,5-dihydroisoxazol-5-yl)phenyl)-7-methyl-2-phenylquinazolin-4(3H)-one (25). Yield: 64\%; m.p. $150-152^{\circ} \mathrm{C}$; TLC solvent (B:A, 9:1); $R_{f}$ 0.62; IR $\left(\mathrm{KBr}, \mathrm{cm}^{-1}\right): 3028(\mathrm{Ar}-\mathrm{CH}), 1692(\mathrm{CO}), 1618(\mathrm{C}=\mathrm{N})$, 1590, $1422(\mathrm{C}=\mathrm{C}), 1222(\mathrm{C}-\mathrm{O}-\mathrm{N}), 1120(\mathrm{C}-\mathrm{N}) ;{ }^{1} \mathrm{H}$ NMR (DMSO-d6) $\delta$ (ppm); $2.12\left(\mathrm{~s}, 3 \mathrm{H}, \mathrm{CH}_{3}\right), 3.80$ (d, 2H, $J=$ $7.2 \mathrm{~Hz}, \mathrm{CH}_{\text {2isox. }}$ ), 5.88 (d, $1 \mathrm{H}, J=4.8 \mathrm{~Hz}, \mathrm{CH}_{\text {isox. }}$ ), $7.92-7.94$ (m, 3H, Ar-H), 8.08-8.10 (m, 5H, Ar-H), $8.12(\mathrm{~d}, 2 \mathrm{H}, J=$ 10.2 Hz, Ar-H), 8.14 (d, 2H, J = 9.2 Hz, Ar-H), 8.33-8.35 (m, $4 \mathrm{H}, \mathrm{Ar}-\mathrm{H}) ;{ }^{13} \mathrm{C}$ NMR (DMSO-d6) $\delta ; 22.5\left(1 \mathrm{C}, \mathrm{CH}_{3}\right), 128.2$ (2C), 128.6, 128.8 (2C), 135.8 (phenyl), 43.0, 83.5, 156.2 (3C, isoxazole), 125.8, 129.0 (2C), 130.8 (2C), 138.8 (Ar-C), 125.8 (2C), 127.8 (2C), 131.2, 138.0 (Ar-C), 118.8, 122.7, 127.5, 130.6, $137.4,152.5,156.4$ (quinazoline), 162.3 (1C, $\mathrm{C}=\mathrm{O}$ ); \% Anal. Cal. for $\mathrm{C}_{30} \mathrm{H}_{22} \mathrm{ClN}_{3} \mathrm{O}_{2} ; \mathrm{C}, 73.24 ; \mathrm{N}, 8.54 ; \mathrm{H}, 4.51$. Found; $\mathrm{C}$, 73.22; N, 8.52; H, 4.50; Ms $(m / z): 491.96\left(\mathrm{M}^{+}\right)$.

(26) 2-(2-Chlorophenyl)-3-(4-(3-(4-chlorophenyl)-4,5-dihydroisoxazol-5-yl)phenyl)-7-methylquinazolin-4(3H)-one (26). Yield: $73 \%$; m.p. $185-187^{\circ} \mathrm{C}$; TLC solvent (B:A, 9:1); $R_{f}$ 0.54; IR (KBr, cm $\left.{ }^{-1}\right): 3040(\mathrm{Ar}-\mathrm{CH}), 1682(\mathrm{CO}), 1622(\mathrm{C}=\mathrm{N})$, 1596, $1424(\mathrm{C}=\mathrm{C}), 1232(\mathrm{C}-\mathrm{O}-\mathrm{N}), 1112(\mathrm{C}-\mathrm{N}), 720(\mathrm{C}-\mathrm{Cl})$; ${ }^{1} \mathrm{H}$ NMR (DMSO-d6) $\delta$ (ppm); $2.34\left(\mathrm{~s}, 3 \mathrm{H}, \mathrm{CH}_{3}\right), 3.89$ (d, $\left.2 \mathrm{H}, J=8.2 \mathrm{~Hz}, \mathrm{CH}_{2 \text { isox. }}\right), 5.93\left(\mathrm{~m}, 1 \mathrm{H}, \mathrm{CH}_{\text {isox. }}\right), 7.94-7.96(\mathrm{~m}$, 
$3 \mathrm{H}, \mathrm{Ar}-\mathrm{H}), 8.10$ (d, 2H, $J=11.2 \mathrm{~Hz}, \mathrm{Ar}-\mathrm{H}), 8.13$ (d, $2 \mathrm{H}, J$ $=2.8 \mathrm{~Hz}, \mathrm{Ar}-\mathrm{H}), 8.14-8.16(\mathrm{~m}, 4 \mathrm{H}, \mathrm{Ar}-\mathrm{H}), 8.20-8.24(\mathrm{~m}$, $4 \mathrm{H}, \mathrm{Ar}-\mathrm{H}) ;{ }^{13} \mathrm{C}$ NMR (DMSO-d6) $\delta ; 20.8\left(1 \mathrm{C}, \mathrm{CH}_{3}\right), 128.6$ (2C), 129.6, 130.2 (2C), 136.2 (phenyl), 42.6, 84.2, 155.8 (3C, isoxazole), 123.4, 126.6, 130.4, 131.8, 132.2, 132.8 (Ar-C), 124.5 (2C), 126.2 (2C), 130.6, 137.6 (Ar-C), 118.8, 124.7, 128.5, 132.6, 143.5, 151.0, 157.2 (quinazoline), 162.3 (1C, $\mathrm{C}=\mathrm{O})$; \% Anal. Cal. for $\mathrm{C}_{30} \mathrm{H}_{21} \mathrm{Cl}_{2} \mathrm{~N}_{3} \mathrm{O}_{2} ; \mathrm{C}, 68.45 ; \mathrm{N}, 7.98 ; \mathrm{H}, 4.02$. Found; C, 68.43; N, 7.96; H, 4.00; Ms (m/z): $528.41(\mathrm{M}+2)$.

(27) 3-(4-(3-(4-Chlorophenyl)-4, 5-dihydroisoxazol-5-yl)phenyl)-7-methyl-2-p-tolylquinazolin-4(3H)-one (27). Yield: 64\%; m.p. 176-178 ${ }^{\circ}$; TLC solvent (B:A, $9: 1$ ); $R_{f} 0.74$; IR $\left(\mathrm{KBr}, \mathrm{cm}^{-1}\right)$ : 3030 (Ar-CH), 1692 (CO), $1614(\mathrm{C}=\mathrm{N}), 1588$, $1420(\mathrm{C}=\mathrm{C}), 1225(\mathrm{C}-\mathrm{O}-\mathrm{N}), 1130(\mathrm{C}-\mathrm{N}), 708(\mathrm{C}-\mathrm{Cl}) ;{ }^{1} \mathrm{H}$ NMR (DMSO-d6) $\delta$ (ppm); 2.20 (s, 3H, $\left.\mathrm{CH}_{3}\right), 2.24$ (s, 3H, $\left.\mathrm{CH}_{3}\right), 3.84\left(\mathrm{~d}, 2 \mathrm{H}, J=2.8 \mathrm{~Hz}, \mathrm{CH}_{2 \text { isox. }}\right), 5.90(\mathrm{~d}, 1 \mathrm{H}, J=$ $8.2 \mathrm{~Hz}, \mathrm{CH}_{\text {isox. }}$ ), 7.88-7.90 (m, 3H, Ar-H), 7.97-7.98 (m, 4H, Ar-H), 8.10 (d, $2 \mathrm{H}, J=6.4 \mathrm{~Hz}, \operatorname{Ar}-\mathrm{H}), 8.12(\mathrm{~d}, 2 \mathrm{H}, J=8.2 \mathrm{~Hz}$, Ar-H), 8.16-8.18 (m, 4H, Ar-H); ${ }^{13} \mathrm{C}$ NMR (DMSO-d6) $\delta$; 22.5 (2C, $\mathrm{CH}_{3}$ ), 128.0 (2C), 128.8, 129.2 (2C), 136.0 (phenyl), 42.8, 84.2, 156.6 (3C, isoxazole), 125.8, 129.2 (2C), 130.8 (2C), 140.2 (Ar-C), 125.2 (2C), 128.5 (2C), 130.4, 138.8 (Ar-C), 118.2, 120.7, 128.5, 130.2, 142.4, 151.2, 155.6 (quinazoline), 160.5 (1C, $\mathrm{C}=\mathrm{O}$ ); \% Anal. Cal. for $\mathrm{C}_{31} \mathrm{H}_{24} \mathrm{ClN}_{3} \mathrm{O}_{2} ; \mathrm{C}, 73.58 ; \mathrm{N}, 8.30$; $\mathrm{H}, 4.78$. Found; C, 73.56; N, 8.28; H, 4.76; Ms $(\mathrm{m} / \mathrm{z}): 506.99$ $(\mathrm{M}+1)$.

(28) 3-(4-(3-(4-Chlorophenyl)-4, 5-dihydroisoxazol-5-yl) phenyl)-2-(4-methoxyphenyl)-7-methylquinazolin-4(3H)-one (28). Yield: $86 \%$; m.p. $122-124^{\circ} \mathrm{C}$; TLC solvent (B:A, $9: 1$ ); $R_{f}$ 0.62; IR $\left(\mathrm{KBr}, \mathrm{cm}^{-1}\right): 3036(\mathrm{Ar}-\mathrm{CH}), 1687$ (CO), 1624 $(\mathrm{C}=\mathrm{N}), 1586,1416(\mathrm{C}=\mathrm{C}), 1288(\mathrm{C}-\mathrm{O}), 1224(\mathrm{C}-\mathrm{O}-\mathrm{N}), 1104$ $(\mathrm{C}-\mathrm{N}), 732(\mathrm{C}-\mathrm{Cl}) ;{ }^{1} \mathrm{H}$ NMR (DMSO-d6) $\delta$ (ppm); 2.19 $\left(\mathrm{s}, 3 \mathrm{H}, \mathrm{CH}_{3}\right), 2.96\left(\mathrm{~s}, 3 \mathrm{H}, \mathrm{OCH}_{3}\right), 3.96(\mathrm{~d}, 2 \mathrm{H}, J=8.0 \mathrm{~Hz}$, $\left.\mathrm{CH}_{2 \text { isox. }}\right), 5.78\left(\mathrm{~m}, 1 \mathrm{H}, \mathrm{CH}_{\text {isox. }}\right), 8.00-8.02(\mathrm{~m}, 3 \mathrm{H}, \mathrm{Ar}-\mathrm{H})$, 8.04-8.06 (m, 4H, Ar-H), 8.08 (d, 2H, $J=5.2 \mathrm{~Hz}$, Ar-H), $8.12(\mathrm{~d}, 2 \mathrm{H}, \mathrm{J}=8.0 \mathrm{~Hz}, \mathrm{Ar}-\mathrm{H}), 8.13-8.15(\mathrm{~m}, 4 \mathrm{H}, \mathrm{Ar}-\mathrm{H}) ;{ }^{13} \mathrm{C}$ NMR (DMSO-d6) $\delta$; $20.2\left(1 \mathrm{C}, \mathrm{CH}_{3}\right), 53.8\left(1 \mathrm{C}, \mathrm{OCH}_{3}\right), 128.2$ (2C), 128.7129 .8 (2C), 135.5 (phenyl), 42.4, 85.7, 155.8 (3C, isoxazole), 115.2 (2C), 120.2, 131.2 (2C), 162.5 (Ar-C), 125.6 (2C), 128.8 (2C), 131.4, 137.4 (Ar-C), 117.0, 124.7, 127.5, 130.5, 143.5, 150.2, 156.2 (quinazoline), 161.8 (1C, $\mathrm{C}=\mathrm{O})$; \% Anal. Cal. for $\mathrm{C}_{31} \mathrm{H}_{24} \mathrm{ClN}_{3} \mathrm{O}_{3}$; C, 71.33; N, 8.05; H, 4.63. Found; $\mathrm{C}$, 71.31; N, 8.03; H, 4.65; Ms (m/z): $521.99\left(\mathrm{M}^{+}\right)$.

(29) 7-Chloro-3-(4-(3-(4-methoxyphenyl)-4,5-dihydroisoxazol-5-yl)phenyl)-2-phenylquinazolin-4(3H)-one (29). Yield: 65\%; m.p. 190-192 C; TLC solvent (B:A, 9:1); $R_{f}$ 0.75; IR $\left(\mathrm{KBr}, \mathrm{cm}^{-1}\right)$ : 3040 (Ar-CH), 1680 (CO), $1614(\mathrm{C}=\mathrm{N}), 1595$, $1417(\mathrm{C}=\mathrm{C}), 1308(\mathrm{C}-\mathrm{O}), 1230(\mathrm{C}-\mathrm{O}-\mathrm{N}), 1120(\mathrm{C}-\mathrm{N}), 722$ $(\mathrm{C}-\mathrm{Cl}) ;{ }^{1} \mathrm{H}$ NMR (DMSO-d6) $\delta$ (ppm); 2.78 (s, 3H, $\mathrm{OCH}_{3}$ ), $3.85\left(\mathrm{~d}, 2 \mathrm{H}, J=4.6 \mathrm{~Hz}, \mathrm{CH}_{2 \text { isox. }}\right), 5.80(\mathrm{~d}, 1 \mathrm{H}, J=8.2 \mathrm{~Hz}$, $\mathrm{CH}_{\text {isox. }}$ ), 8.06-8.08 (m, 4H, Ar-H), 8.10 (d, 2H, $J=8.8 \mathrm{~Hz}$, Ar-H), $8.12(\mathrm{~d}, 2 \mathrm{H}, J=6.0 \mathrm{~Hz}, \operatorname{Ar}-\mathrm{H}), 8.13-8.15(\mathrm{~m}, 3 \mathrm{H}$, Ar-H), 8.16-8.18 (m,5H, Ar-H); ${ }^{13} \mathrm{C}$ NMR (DMSO-d6) $\delta$; 52.0 (1C, $\mathrm{OCH}_{3}$ ), 115.5 (2C), 122.6128 .2 (2C), 160.8 (phenyl), 43.2, 84.7, 156.4 (3C, isoxazole), 128.2 (2C), 128.6, 129.5 (2C),
130.2 (Ar-C), 124.5 (2C), 128.3 (2C), 130.4, 138.2 (Ar-C), 118.6, $122.6,127.8,130.5,138.2,152.8,157.7$ (quinazoline), 160.4 (1C, $\mathrm{C}=\mathrm{O}$ ); \% Anal. Cal. for $\mathrm{C}_{30} \mathrm{H}_{22} \mathrm{ClN}_{3} \mathrm{O}_{3} ; \mathrm{C}, 70.93 ; \mathrm{N}, 8.27$; $\mathrm{H}$, 4.37. Found; C, 70.95; N, 8.25; H, 4.35; Ms $(\mathrm{m} / z): 507.96$ $\left(\mathrm{M}^{+}\right)$.

(30) 7-Chloro-2-(2-chlorophenyl)-3-(4-(3-(4-methoxyphenyl)4, 5-dihydroisoxazol-5-yl)phenyl)quinazolin-4(3H)-one (30). Yield: $86 \%$; m.p. $179-181^{\circ}$ C; TLC solvent (B : A, $\left.9: 1\right) ; R_{f} 0.64$; IR $\left(\mathrm{KBr}, \mathrm{cm}^{-1}\right): 3035$ (Ar-CH), $1696(\mathrm{CO}), 1620(\mathrm{C}=\mathrm{N}), 1598$, 1430 (C=C), 1278 (C-O), $1234(\mathrm{C}-\mathrm{O}-\mathrm{N}), 1134(\mathrm{C}-\mathrm{N}), 722$ $(\mathrm{C}-\mathrm{Cl}) ;{ }^{1} \mathrm{H}$ NMR (DMSO-d6) $\delta$ (ppm); 2.82 (s, 3H, $\mathrm{OCH}_{3}$ ), $3.83\left(\mathrm{~d}, 2 \mathrm{H}, J=8.4 \mathrm{~Hz}, \mathrm{CH}_{2 \text { isox. }}\right), 5.94\left(\mathrm{~m}, 1 \mathrm{H}, \mathrm{CH}_{\text {isox. }}\right), 7.96-$ 7.98 (m, 4H, Ar-H), 8.00-8.02 (m, 3H, Ar-H), 8.02-8.04 (m, $4 \mathrm{H}, \mathrm{Ar}-\mathrm{H}), 8.08$ (d, 2H, J = 6.2 Hz, Ar-H), 8.10 (d, 2H, $J=$ $9.4 \mathrm{~Hz}, \mathrm{Ar}-\mathrm{H}) ;{ }^{13} \mathrm{C}$ NMR (DMSO-d6) $\delta ; 55.9\left(1 \mathrm{C}, \mathrm{OCH}_{3}\right)$, 116.4 (2C), 122.5128 .2 (2C), 160.4(phenyl), 43.4, 84.6, 156.2 (3C, isoxazole), 122.5, 126.8, 130.5, 131.6, 132.2, 132.8 (Ar-C), 125.0 (2C), 127.6 (2C), 131.8, 138.9 (Ar-C), 118.6, 122.5, 128.2, $131.5,137.8,151.6,155.8$ (quinazoline), 160.2 (1C, $\mathrm{C}=\mathrm{O}) ; \%$ Anal. Cal. for $\mathrm{C}_{30} \mathrm{H}_{21} \mathrm{Cl}_{2} \mathrm{~N}_{3} \mathrm{O}_{3} ; \mathrm{C}, 66.43 ; \mathrm{N}, 7.75 ; \mathrm{H}, 3.90$. Found; C, 66.41; N, 7.73; H, 3.92; Ms $(m / z)$ : $544.41(\mathrm{M}+2)$.

3.2. Pharmacology. All the experimental protocols were carried out with the permission from the Institutional Animal Ethics committee (IAEC), project proposal no. 781 and the guidelines provided by the Committee for the Purpose of Control and Supervision of Experiments in Animal (CPCSEA). Animals were obtained from the Central Animal House Facility, Hamdard University, New Delhi. Registration number and date of registration are 173/CPCSEA, 28th of January, 2000. All rats were housed in a temperature and humidity controlled room at an ambient temperature of $25 \pm$ $2^{\circ} \mathrm{C}$ with a $12 \mathrm{~h}$ light/dark cycle. Animals were provided with pellets diet (Lipton, Calcutta, India) and water ad libitum.

\subsubsection{Antihypertensive Activity}

3.2.2. Conditioning/Training of Animals. For conducting the BP measurement studies, the animals were kept in restrainers for $10 \mathrm{~min}$ every day for one week. This exercise was done to avoid the fluctuation in blood pressure due to aggressive behaviour of the animal.

3.2.3. Induction of Hypertension in Albino Rats. After recording the initial BP of rats, the animals were divided into groups of 6 animals each. One group was taken as control. Hypertension was induced in the remaining groups by subcutaneous injection of methyl prednisolone acetate $(20 \mathrm{mg} / \mathrm{kg}$ body wt./wk) for 2 weeks as per method reported by Krakoff et al. [19].

3.2.4. Dose Establishment of Synthesized Compounds for Screening of Antihypertensive Activity. Systolic blood pressure was measured in conscious rats using CODA noninvasive blood pressure recorder by Tail Cuff method (Kent Scientific Corporation, USA). The restrainers carrying the rat were placed in the BP instrument with the tail protruding 
out. The tail was gently placed in contact with a transducer membrane, which was connected to the digital BP display panel. The instrument was then turned on and allowed to stabilize until steady pulse rate was observed. Once the "pulse level ready" signal appeared, the BP recording button was pressed and the mean arterial BP was recorded. Albino rats (body weight 200-250 g) were used in the study. Rats were assigned to groups of six animals in each. Each compound (1 mg/day, $2 \mathrm{mg} /$ day,. ..,10 mg/day) was administered orally after suspending in $1 \%$ carboxy methyl cellulose (CMC) solution. The blood pressure was recorded at various time intervals.

\subsubsection{Determination of $\alpha$-Adrenergic Receptors Blocking Activ-} ity. Albino rats were classified into ten groups; each group comprises 6 animals each, to investigate the $\alpha_{1}$-adrenergic receptor blocking effect of some synthesized compounds. In each experiment, the effect of adrenaline, at a dose of $3 \mu \mathrm{g} / \mathrm{Kg}$ intravenously, on the arterial blood pressure was recorded alone $30 \mathrm{~min}$ before i.p. injection $(5 \mathrm{mg} / \mathrm{kg}$ b.w. for compounds $1,16,19,20,23,27$, and 28 , whereas $4 \mathrm{mg} / \mathrm{kg}$ b.w. for compound 24) of the test compound and then its effect was determined again after 30, 60, and $120 \mathrm{~min}$ from the injection of the test compounds [20].

3.2.6. Statistical Analysis of Data. The statistical analysis was performed using GRAPHPAD INSTAT 3 software (Graph Pad Software Inc., San Diego, CA). Data obtained from animal experiments were expressed as arithmetic mean \pm $S E M$ and \pm S.E. The comparison between various groups was performed by one-way analysis of variance (ANOVA), and the effects in treatment groups were compared with toxic control or control group by Dunnett multiple comparison test. $P<0.05$ was considered to be significant $\left({ }^{*} P<0.05\right.$; $\left.{ }^{* *} P<0.01\right)$.

\section{Result and Discussions}

4.1. Chemistry. 7-Substituted-3-(4-(3-(4-substitutedphenyl)4,5-dihydroisoxazol-5-yl)phenyl)-2-substituted quinazolin$4(3 \mathrm{H})$-one derivatives (1-30) were synthesized according to Scheme 1. Substituted anthranilic acids were reacted with substituted benzoyl chloride via stirring the reactants at $10 \%$ $\mathrm{NaOH}$ to yield substituted amides (a1-a12). Some substituted benzoxazines (b1-b12) were obtained by heating (a1-a12) at reflux in acetic anhydride [21-25]. Substituted 3-(4acetylphenyl)-2-phenylquinazolin-4(3H)-one (c1-c12) were yielded by the refluxing of substituted benzoxazines with $p$-aminoacetophenone $[26,27]$. According to the ClaisenSchmidt condensation, substituted 3-(4-acetylphenyl)-2phenylquinazolin-4(3H)-one with aromatic aldehydes in equimolar amount were stirred in ethanol for $2 \mathrm{~h}$ to yield different chalcones [28]. Finally, different chalcones and hydroxylamine hydrochloride were heated on a water bath in ethanol containing $\mathrm{KOH}$ to obtain title compounds (1-30) [29]. The purity of all the target compounds was ensured using thin-layer chromatography (TLC) in different solvent systems and melting point techniques and was further confirmed by both the analytical and spectral data of the proposed structures. For instance, for compound 24, The IR spectra of mentioned molecule showed characteristic absorption bands at $3036 \mathrm{~cm}^{-1}$ (Ar-CH), $1680 \mathrm{~cm}^{-1}$ (CO), $1622 \mathrm{~cm}^{-1} \quad(\mathrm{C}=\mathrm{N}), \quad 1596,1420 \mathrm{~cm}^{-1} \quad(\mathrm{C}=\mathrm{C}), \quad 1300 \mathrm{~cm}^{-1}$, $1233 \mathrm{~cm}^{-1}(\mathrm{C}-\mathrm{O}-\mathrm{N}), 1130 \mathrm{~cm}^{-1}(\mathrm{C}-\mathrm{N})$, and $735 \mathrm{~cm}^{-1}(\mathrm{C}-\mathrm{Cl})$. The ${ }^{1} \mathrm{H}$ NMR spectra showed two doublet at $\delta 8.14(2 \mathrm{H}, J=$ $8.0 \mathrm{~Hz}, \mathrm{Ar}-\mathrm{H})$ and $8.18(2 \mathrm{H}, J=4.8 \mathrm{~Hz}, \mathrm{Ar}-\mathrm{H})$ confirming $\mathrm{N}_{2}$-Ar ring, one doublet at $\delta 3.84\left(\mathrm{~d}, 2 \mathrm{H}, J=8.0 \mathrm{~Hz}, \mathrm{CH}_{2 \text { isox. }}\right)$ confirming $\mathrm{CH}_{2}$ isoxazole, and one multiplet at $\delta 6.01$ confirming $\mathrm{CH}$ isoxazole. The multiplets at $\delta 8.00-7.98$, 8.06-8.04, and 8.10-8.08 are indicatives of 4 protons of $\mathrm{Ar}_{2}$ ring, 3 protons of quinazoline moiety, and 4 protons of $\mathrm{N}_{3}$-Ar ring, respectively. The one singlet corresponding to $\mathrm{OCH}_{3}$ was obtained at $\delta$ 2.65. The mass spectrum shows the presence of molecular ion peak at $m / z 544.11(\mathrm{M}+2)$ according to the molecular formula, $\mathrm{C}_{30} \mathrm{H}_{21} \mathrm{Cl}_{2} \mathrm{~N}_{3} \mathrm{O}_{3}$. The structure was also supported by elemental analysis data which was $\pm 0.4 \%$. The other compounds are also characterized in a similar manner.

\subsection{Antihypertensive Activity}

4.2.1. Dose Establishment for the Screening of Antihypertensive Activity. The minimum effective dose of test compounds was established by performing the gradual enhanced dose administration ( $1 \mathrm{mg} /$ day, $2 \mathrm{mg} /$ day, $3 \mathrm{mg} /$ day,. .., $10 \mathrm{mg} /$ day), and when there was no further decrease, that is, ceiling effect in the blood pressure, observed at a certain dose, that dose was assumed as the minimum dose required to elicit the optimum antihypertensive activity of the synthesised compound. All titled compounds were screened to study their effect on the arterial blood pressure by using noninvasive Tail Cuff method. The results are compared with standard drug prazosin [30], as shown in Table 1. Most of the synthetic compounds showed the ceiling dose between 5-6 mg. The title compounds $2,18,19,23$, and 27 were found to show substantial reduction in systolic blood pressure, whereas compound 24 showed significant antihypertensive activity as compared to standard drug prazosin (Figure 2). The ceiling dose of compound $\mathbf{2 4}$ was found to be $4 \mathrm{mg}$; however, prazosin has a ceiling dose of $5 \mathrm{mg}$. The rest of the compounds showed moderate decrease in hypertension.

4.2.2. Determination of $\alpha$-Adrenergic Receptor Blocking Property. The injection of prazosin, $30 \mathrm{~min}$ prior to i.v injection of adrenaline at a dose of $3 \mu \mathrm{g} / \mathrm{Kg}$ [31], produced a significant $(P<0.05)$ drop in arterial blood pressure due to selective blockade of $\alpha_{1}$-adrenergic receptor by prazosin (adrenaline antagonist) [11]; however, injection of prazosin, $60 \mathrm{~min}$ prior to adrenaline injection returned the blood pressure to normal level; this effect may be due to rapid onset and short duration of action of drug (Figures 3 and 4). Amongst the tested compounds, compound 1 showed the significant $(P<0.05)$ increase in SABP and $\mathrm{DABP}$ and without changing heart rate at $30 \mathrm{~min}$ prior 


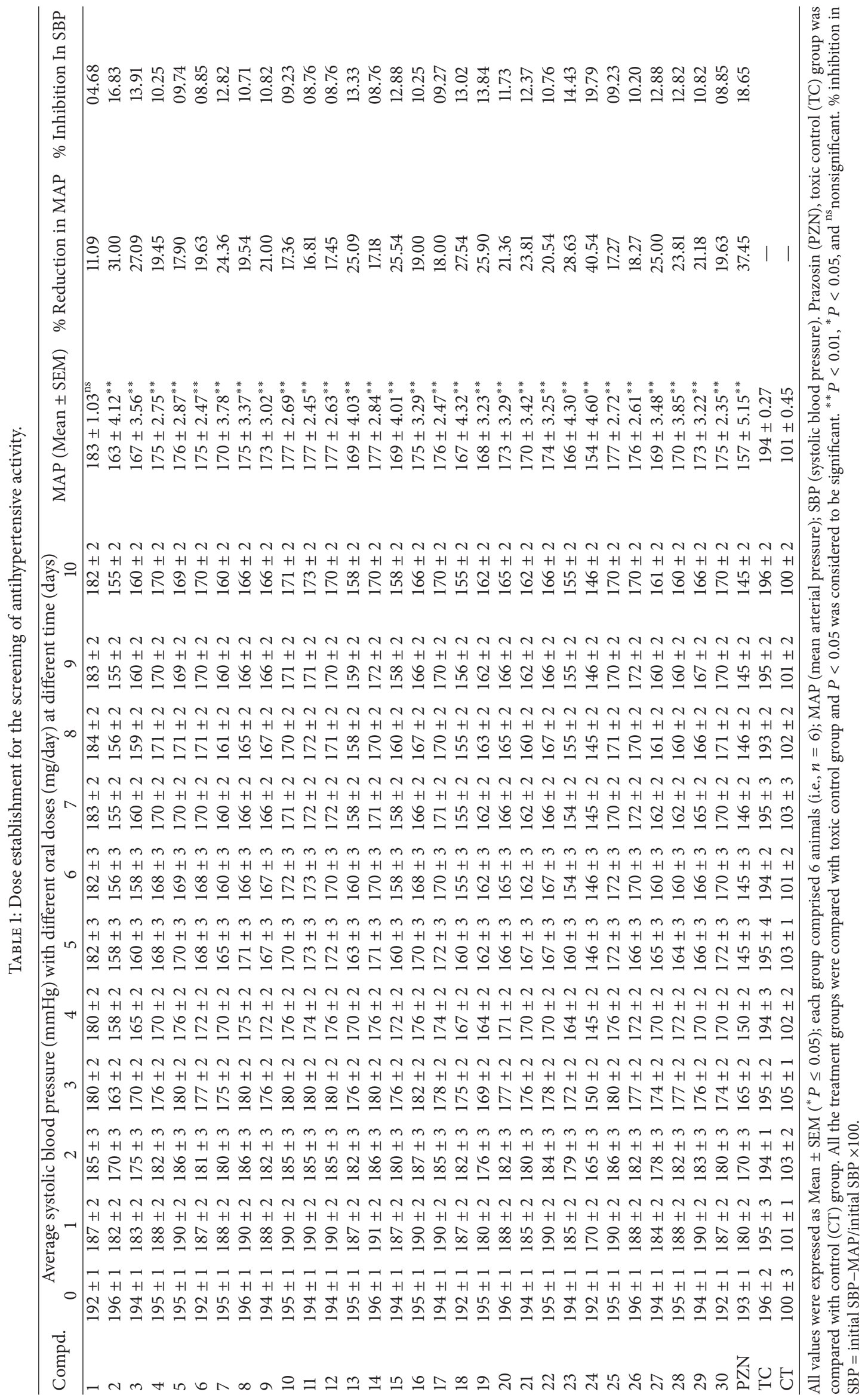


<smiles>[Y1]c1ccc(-c2nc3c([R])cccc3c(=O)n2-c2ccc(C(=O)/C=C/c3ccccc3)cc2)cc1</smiles>

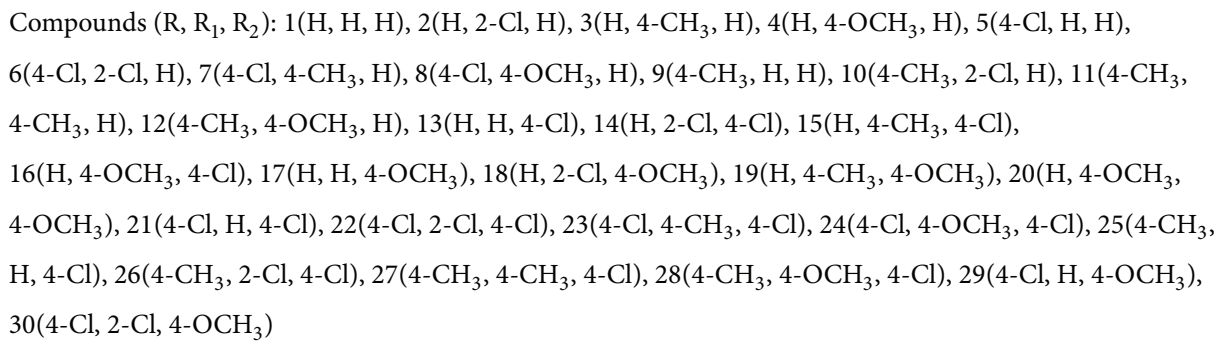

SCHEME 1: Synthesis of 7-substituted-3-(4-(3-(4-substitutedphenyl)-4,5-dihydroisoxazol-5-yl)phenyl)-2-substituted quinazolin-4(3H)-one. Reagents and conditions: (a) $10 \% \mathrm{NaOH}, \mathrm{rt}, 60 \mathrm{~min}$; (b) reflex for $2 \mathrm{~h}$; (c) methanol reflux for $3 \mathrm{~h}$; (d) ethanol, $\mathrm{NaOH}$, stirring at rt, $2 \mathrm{~h}$; (e) $\mathrm{NH}_{2} \mathrm{OH} \cdot \mathrm{HCl}, 30 \% \mathrm{KOH}$, ethanol, dist. water, at water bath, $5 \mathrm{~h}$.

to administration of adrenaline. Therefore this compound could serve as lead molecule for the synthesis of a series of compounds to treat hypotension. Compounds 23 and 24 showed reverse action of vasopressor effect of adrenaline with depressor reflex after $30 \mathrm{~min}$, as shown in Table 2. This effect was further prolonged at $60 \mathrm{~min}$ (Figures 3 and 4). The activity was continued up to $120 \mathrm{~min}$ to check the further change in activity. On the basis of these observations, it may be hypothesized that these compounds possess rapid and prolonged $\alpha_{1}$-adrenergic blocking property without causing reflex tachycardia. Compounds 16, 27, and 28 also exhibited moderate antagonist activity of adrenaline after $30 \mathrm{~min}$. It indicates that these compounds also possess $\alpha_{1}$-adrenergic receptor blocking effect with shorter duration of action like prazosin.

\section{Conclusion}

In conclusion, we have synthesized new quinazoline derivatives associated with isoxazole. These compounds showed significant antihypertensive activity with minimal side effects. The most potent $\alpha_{1}$-adrenergic blocking property was exhibited by the compound 24, having an SABP \& DABP $11.6,-12.6$ at $30 \mathrm{~min}$. The replacement of piperazine moiety 


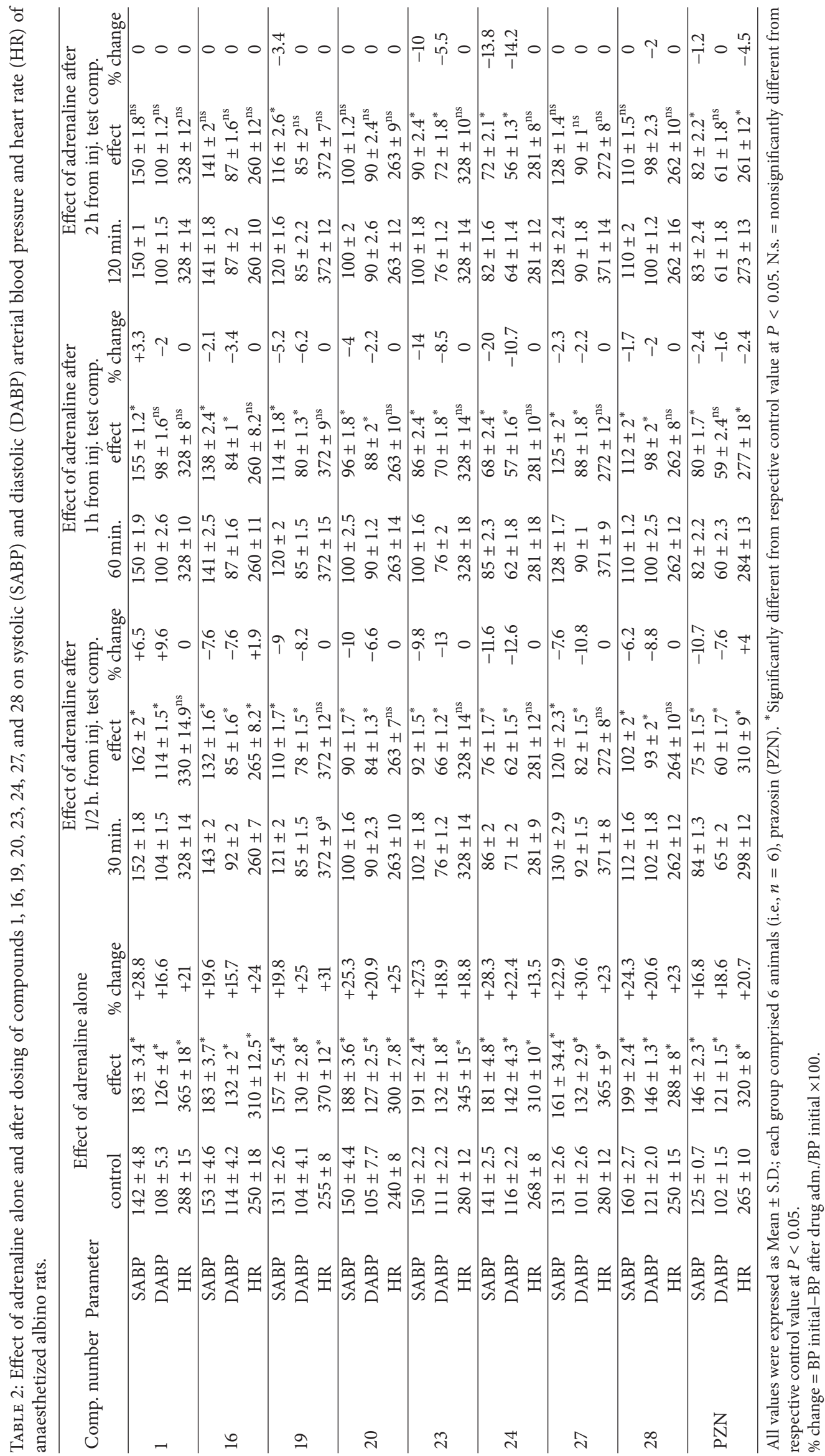




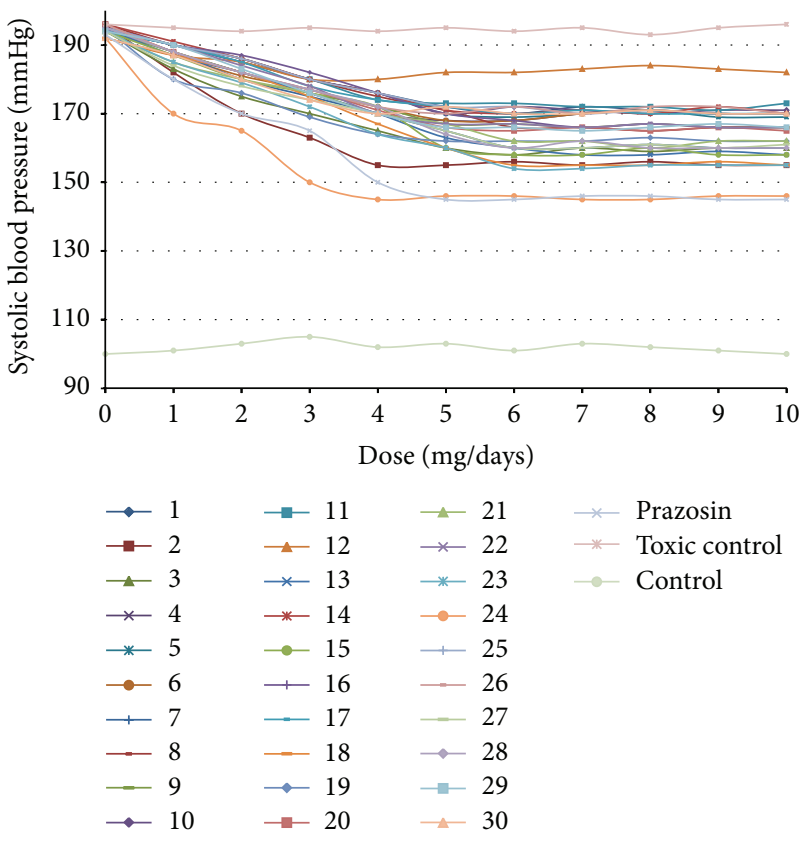

FIGURE 2: Graph showing dose establishment for the screening of antihypertensive activity.

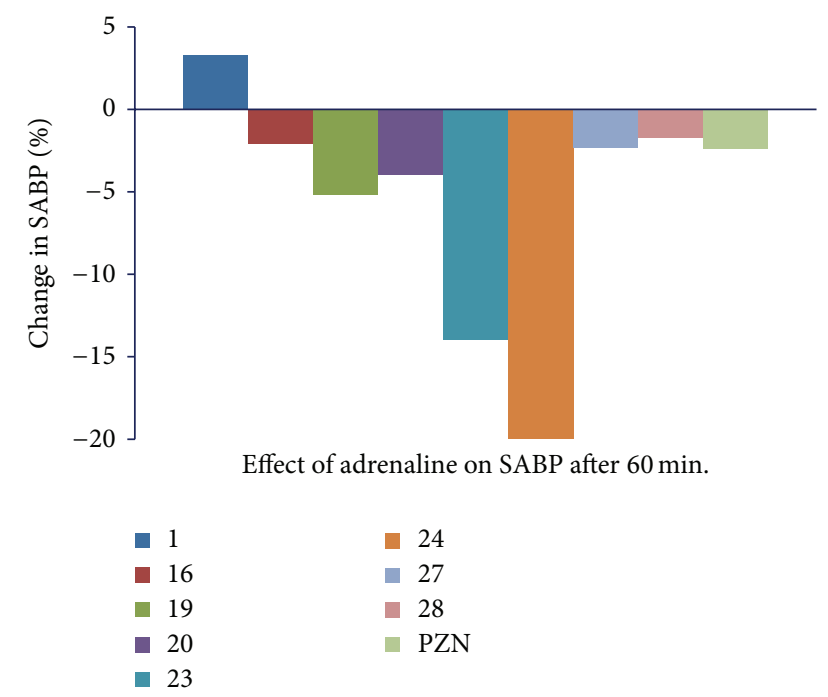

FIGURE 3: Graph showing effect of i.v. inj. of adrenaline $(3 \mu \mathrm{g} / \mathrm{Kg})$ $30 \mathrm{~min}$ after prazosin and some test compounds 1, 16, 19, 20, 23, 24,27 , and 28 at a dose of $5 \mathrm{mg} / \mathrm{Kg}$ on systolic blood pressure of anaesthetized albino rats.

of prazosin at position-2 with substituted phenyl ring and incorporation of substituted isoxazole ring at position-3 of quinazoline moiety proved beneficial for antihypertensive activity. Our study shows that these synthesized compounds act as lead and may be useful in antihypertensive therapy.

\section{Disclaimer}

The authors alone are responsible for the content and writing of the paper.

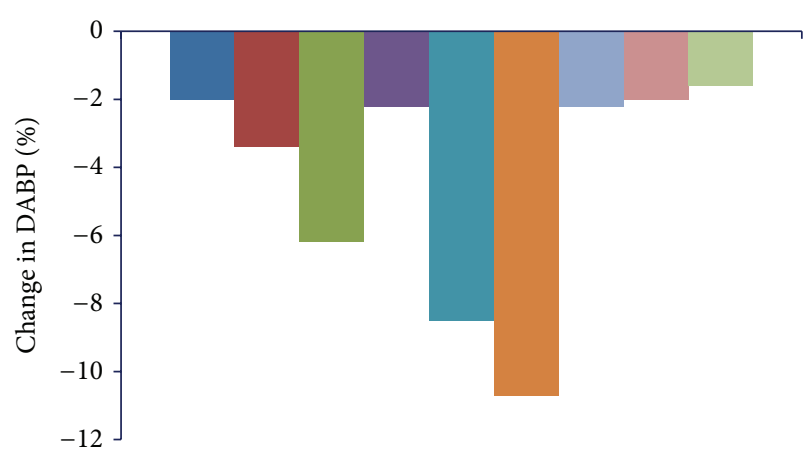

Effect of adrenaline on DABP after $60 \mathrm{~min}$.

$\begin{array}{ll}1 & \\ \square & 24 \\ 16 & \\ 19 & 27 \\ - & 28 \\ \square & \\ 23 & \mathrm{PZN}\end{array}$

FIGURE 4: Graph showing effect of i.v. inj. of adrenaline $(3 \mu \mathrm{g} / \mathrm{Kg})$ 30 min after prazosin and some test compounds 1, 16, 19, 20, 23, 24,27 , and 28 at a dose of $5 \mathrm{mg} / \mathrm{Kg}$ on diastolic blood pressure of anaesthetized albino rats.

\section{Conflict of Interests}

The authors report no conflict of interests.

\section{Acknowledgments}

The authors are thankful to Jamia Hamdard, New Delhi (India), for providing the research facilities. Mujeeb Ur Rahman is also thankful to UGC (MANF) for providing financial assistance.

\section{References}

[1] A. Ferro, R. Gilbert, and H. Krum, "Importance of renin in blood pressure regulation and therapeutic potential of renin inhibition," International Journal of Clinical Practice, vol. 60, no. 5, pp. 577-581, 2006.

[2] "American Heart Association," Statistics, 2007.

[3] P. M. Kearney, M. Whelton, K. Reynolds, P. Muntner, P. K. Whelton, and J. He, "Global burden of hypertension: analysis of worldwide data," The Lancet, vol. 365, no. 9455, pp. 217-223, 2005.

[4] J. Cook, K. D. Frick, and R. Baltussen, "Loss of vision and hearing," in Disease Control Priorities in Developing Countries, D. T. Jamison, J. G. Breman, and A. R. Measham, Eds., chapter 50, pp. 953-962, Oxford University Press, New York, NY, USA, 2nd edition, 2006.

[5] A. Leonardi, G. Motta, C. Boi et al., "Synthesis, pharmacological evaluation, and structure-activity relationship and quantitative structure-activity relationship studies on novel derivatives of 2,4-diamino-6,7-dimethoxyquinazoline $\alpha 1$ adrenoceptor antagonists," Journal of Medicinal Chemistry, vol. 42, no. 3, pp. 427-437, 1999.

[6] P. Karczewski, H. Haase, P. Hempel, and M. Bimmler, "Agonistic antibody to the $\alpha 1$-adrenergic receptor mobilizes intracellular calcium and induces phosphorylation of a cardiac $15-\mathrm{kDa}$ 
protein," Molecular and Cellular Biochemistry, vol. 333, no. 1-2, pp. 233-242, 2010.

[7] N. A. Colabufo, V. Pagliarulo, F. Berardi et al., "Human epididymal and prostatic tracts of vas deferens: different contraction response to noradrenaline stimulation in isolated organ bath assay," European Journal of Pharmacology, vol. 577, no. 1-3, pp. 150-155, 2007.

[8] B. V. Shetty, L. A. Campanella, T. L. Thomas et al., "Synthesis and activity of some 3-aryl- and 3-aralkyl-1,2,3,4-tetrahydro-4-oxo6-quinazolinesulfonamides," Journal of Medicinal Chemistry, vol. 13, no. 5, pp. 886-895, 1970.

[9] F. Carta and C. T. Supuran, "Diuretics with carbonic anhydrase inhibitory action: a patent and literature review (2005-2013)," Expert Opinion on Therapeutic Patents, vol. 23, no. 6, pp. 681691, 2013.

[10] D. Ekinci, N. I. Kurbanoglu, E. Salamci, M. Şentürk, and C. T. Supuran, "Carbonic anhydrase inhibitors: inhibition of human and bovine isoenzymes by benzenesulphonamides, cyclitols and phenolic compounds," Journal of Enzyme Inhibition and Medicinal Chemistry, vol. 27, no. 6, pp. 845-848, 2012.

[11] O. I. El-Sabbagh, M. A. Shabaan, H. H. Kadry, and E. S. Al-Din, "New octahydroquinazoline derivatives: synthesis and hypotensive activity," European Journal of Medicinal Chemistry, vol. 45, no. 11, pp. 5390-5396, 2010.

[12] V. Alagarsamy, R. Venkatesaperumal, S. Vijayakumar et al., "Synthesis and pharmacological investigation of some novel 2phenyl-3-(substituted methyl amino) quinazolin-4(3H)-ones as $\mathrm{H}_{1}$-receptor blockers," Pharmazie, vol. 57, no. 5, pp. 306-307, 2002.

[13] V. Alagarsamy, "Synthesis and pharmacological investigation of some novel 2-methyl-3-(substituted methylamino)-(3H)quinazolin-4-ones as histamine H1-receptor blockers," Pharmazie, vol. 59, no. 10, pp. 753-755, 2004.

[14] V. Alagarsamy, V. R. Salomon, G. Vanikavitha et al., "Synthesis, analgesic, anti-inflammatory and antibacterial activities of some novel 2-phenyl-3-substituted quinazolin-4(3H) ones," Biological and Pharmaceutical Bulletin, vol. 25, no. 11, pp. 14321435, 2002.

[15] V. Alagarsamy, D. Shankar, V. R. Solomon, R. V. Sheorey, and P. Parthiban, "Synthesis and pharmacological evaluation of 3cyclohexyl-2-substituted hydrazino-3H-quinazolin-4-ones as analgesic and anti-inflammatory agents," Acta Pharmaceutica, vol. 59, no. 1, pp. 75-88, 2009.

[16] M. J. Hour, L. J. Huang, S. C. Kuo et al., "6-alkylaminoand 2,3-dihydro-3'-methoxy-2-phenyl-4-quinazolinones and related compounds: their synthesis, cytotoxicity, and inhibition of tubulin polymerization," Journal of Medicinal Chemistry, vol. 43, no. 23, pp. 4479-4487, 2000.

[17] V. Alagarsamy, R. Giridhar, M. R. Yadav, R. Revathi, K. Ruckmani, and E. de Clercq, "AntiHIV, antibacterial and antifungal activities of some novel 1,4-disubstituted-1,2,4-triazolo[4,3-a] quinazolin-5(4H)-ones," Indian Journal of Pharmaceutical Sciences, vol. 68, no. 4, pp. 532-535, 2006.

[18] A. A. Siddiqui, R. Mishra, and M. Shaharyar, "Synthesis, characterization and antihypertensive activity of pyridazinone derivatives," European Journal of Medicinal Chemistry, vol. 45, no. 6, pp. 2283-2290, 2010.

[19] L. R. Krakoff, R. Selvadurai, and E. Sutter, "Effect of methylprednisolone upon arterial pressure and the renin angiotensin system in the rat," The American Journal of Physiology, vol. 228, no. 2, pp. 613-617, 1975.
[20] B. B. Hoffman and B. G. Katzung, Basic and Clinical Pharmacology, Appleton and Lange, Stamford, Conn, USA, 7th edition, 1998.

[21] S. M. Mosaad, K. I. Mohammed, M. A. Ahmed, and S. G. J. Abdel-Hamide, "Synthesis of certain new 6-iodoquinazolines as potential antitubercular agents," Journal of Applied Sciences, vol. 4, no. 2, pp. 302-307, 2004.

[22] G. Grover and S. G. Kini, "Synthesis and evaluation of new quinazolone derivatives of nalidixic acid as potential antibacterial and antifungal agents," European Journal of Medicinal Chemistry, vol. 41, no. 2, pp. 256-262, 2006.

[23] O. M. O. Habib, H. M. Hassan, and A. El-Mekabaty, "Novel quinazolinone derivatives: synthesis and antimicrobial activity," Medicinal Chemistry Research, vol. 22, no. 2, pp. 507-519, 2013.

[24] R. R. Mohan, R. Agarwal, and V. S. Misr, "Synthesis of some newer quinazolinyl-oxadiazoles, thiosemicarbazides and thiadiazoles as pharmacologically active agents," Indian Journal of Chemistry B, vol. 24, pp. 78-82, 1985.

[25] A. Kumar, S. Sharma, K. Bajaj et al., "Some new 2,3,6trisubstituted quinazolinones as potent anti-inflammatory, analgesic and COX-II inhibitors," Bioorganic and Medicinal Chemistry, vol. 11, no. 23, pp. 5293-5299, 2003.

[26] M. A. Sayed, A. F. El-Kafrawy, A. Y. Osman, and F. A. El-Bassiouny, "The chemistry of 4H-3,1-benzoxazin-4-ones," Indian Journal of Chemistry B, vol. 30, p. 980, 1991.

[27] A. R. R. Rao and V. M. Reddy, "New esters of heteryl acetic and mercaptoacetic acids. Synthesis and H1-antihistaminic evaluation," Arzneimittel Forschung/Drug Research, vol. 43, pp. 633-667, 1993.

[28] M. Shaharyar, M. M. Abdullah, M. A. Bakht, and J. Majeed, "Pyrazoline bearing benzimidazoles: search for anticancer agent," European Journal of Medicinal Chemistry, vol. 45, no. 1, pp. 114-119, 2010.

[29] S. B. Lohiya and B. J. Ghiya, "Reaction of a chalcone with hydroxylamine," Indian Journal of Chemistry B, vol. 25, pp. 279282, 1986.

[30] V. Alagarsamy and U. S. Pathak, "Synthesis and antihypertensive activity of novel 3-benzyl-2-substituted-3H-[1,2,4] triazolo[5,1b]quinazolin-9-ones," Bioorganic and Medicinal Chemistry, vol. 15, no. 10, pp. 3457-3462, 2007.

[31] M. N. Ghosh, Fundamentals of Experimental Pharmacology, Scientific Book Agency, Calcutta, India, 1st edition, 1971. 

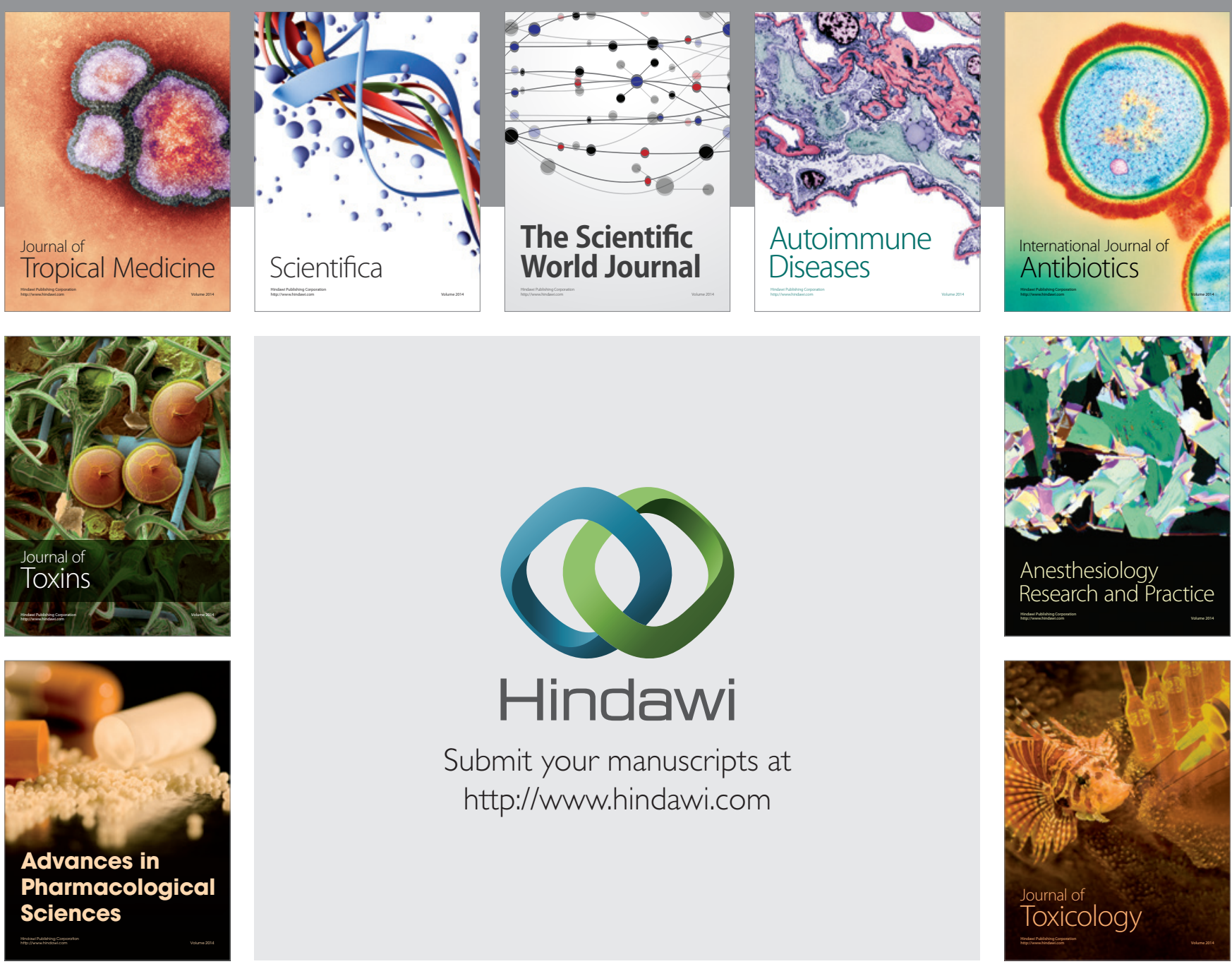

\section{Hindawi}

Submit your manuscripts at

http://www.hindawi.com
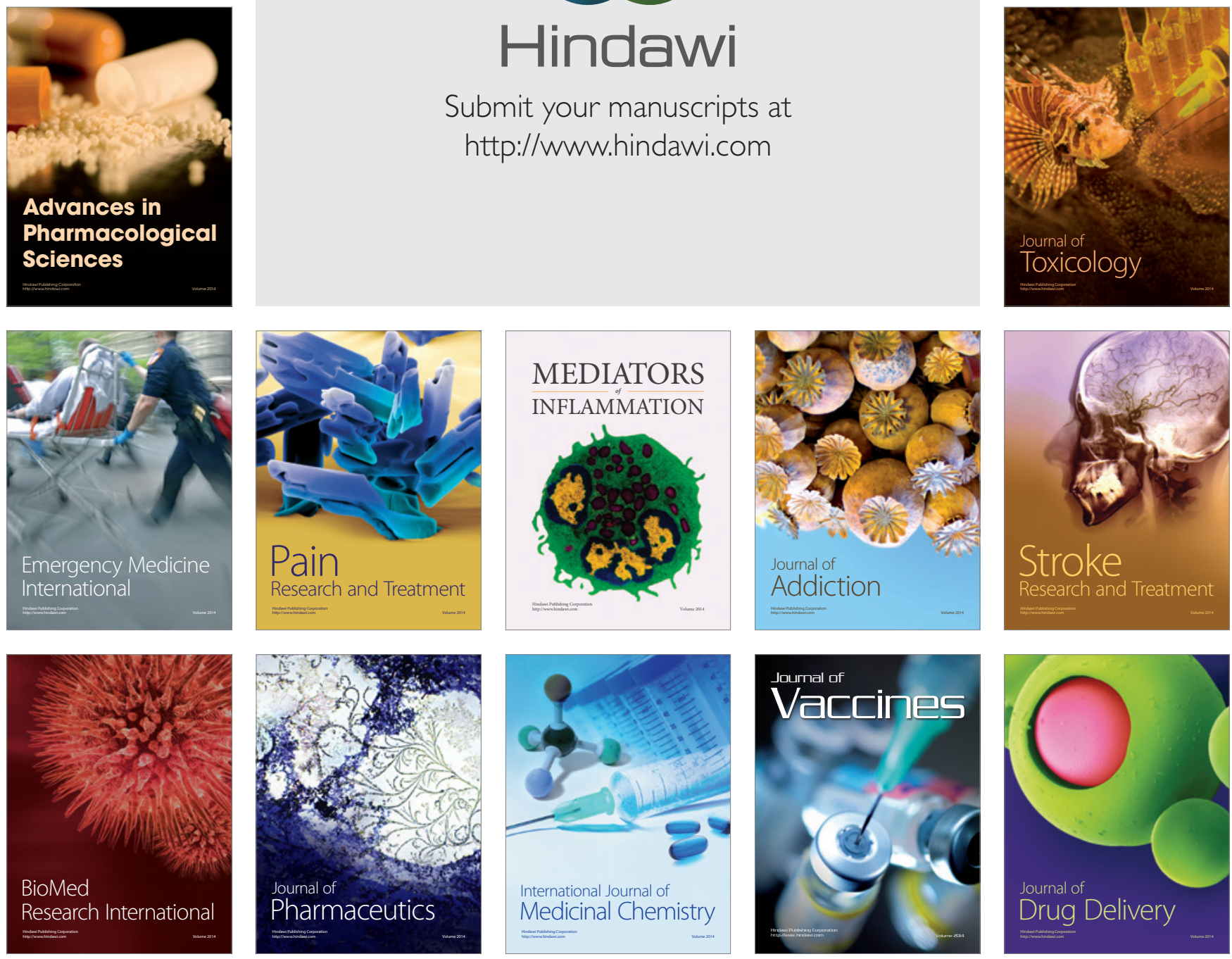